\title{
Time-domain Pure-state Polarization Analysis of Surface Waves Traversing California
}

\author{
Jiajun Zhang, ${ }^{1}$ William R. Walter, ${ }^{2}$ \\ THORNE LAY, ${ }^{1}$ and Ru-SHAN Wu ${ }^{1}$
}

\begin{abstract}
A time-domain pure-state polarization analysis method is used to characterize surface waves traversing California parallel to the plate boundary. The method is applied to data recorded at four broadband stations in California from twenty-six large, shallow earthquakes which occurred since 1988, yielding polarization parameters such as the ellipticity, Euler angles, instantaneous periods, and wave incident azimuths. The earthquakes are located along the circum-Pacific margin and the ray paths cluster into two groups, with great-circle paths connecting stations MHC and PAS or CMB and GSC. The first path (MHC-PAS) is in the vicinity of the San Andreas Fault System (SAFS), and the second (CMB-GSC) traverses the Sierra Nevada Batholith parallel to and east of the SAFS. Both Rayleigh and Love wave data show refractions due to lateral velocity heterogeneities under the path, indicating that accurate phase velocity and attenuation analysis requires array measurements. The Rayleigh waves are strongly affected by low velocity anomalies beneath Central California, with ray paths bending eastward as waves travel toward the south, while Love waves are less affected, providing observables to constrain the depth extent of anomalies. Strong lateral gradients in the lithospheric structure between the continent and the ocean are the likely cause of the path deflections.
\end{abstract}

Key words: Seismic wave polarization, surface waves, refraction.

\section{Introduction}

Travel-time and amplitude measurements of seismic waves play central roles in studies of seismic sources and earth's structure; however accurate measurements may not always be obtained if great-circle propagation is assumed, particularly for surface waves (e.g., Mitchell, 1995). For a spherically symmetric isotropic medium, the particle motions of principle seismic waves (i.e., $P, S$, Rayleigh, and Love waves) are linearly or elliptically polarized parallel to or orthogonal to the great-circle plane which contains the propagation path. However, for a laterally heterogeneous and anisotropic medium like the earth, the particle motion usually involves interference of direct, refracted, reflected, and scattered body and surface waves that are not constrained to a plane, resulting in complex polarization and energy transport off the

\footnotetext{
${ }^{1}$ Institute of Geophysics and Planetary Physics, University of California, Santa Cruz, U.S.A. E-mail: tlay@es.ucsc.edu

${ }^{2}$ L-205, Lawrence Livermore National Laboratory, Livermore, U.S.A.
} 
great-circle. This complexity has motivated the development of sophisticated polarization analysis techniques to constrain the effects of heterogeneity and anisotropy on seismic wave propagation, including path deflection effects (e.g., Booth et al., 1991; CrAmpin, 1970, 1975; CRAMPIN et al., 1990, 1991; KirKwOOD and Crampin, 1981; PARK et al., 1987; Silver and Chan 1988; VinniK et al., 1989; Laske and Masters, 1996; Vig and Mitchell, 1990; Aster et al., 1990, 1991; LEVSHIN et al., 1992).

The effects of the earth's lateral heterogeneity on the propagation of surface waves have been studied extensively (e.g., MCGARR, 1969; CAPON, 1970; BUNGUM and CAPON, 1974; PAtTon, 1980; LAy and KanAmori, 1985; Jobert and Jobert, 1983; Woodhouse and Wong, 1986; Lerner-Lam and PARK, 1989; LeVshin et al., 1992). Surface wave amplitude and travel-time anomalies are often associated with polarization anomalies and involve horizontal refraction caused by lateral heterogeneities. Various time or frequency domain correlation and filtering techniques are commonly used in surface wave polarization analysis (e.g., ArCHAMBEAU et al., 1965; Montalbetti and KanASEWICH, 1970; LeVShin et al., 1972; KANASEWICH, 1981; Vidale 1986; PARK et al., 1987; JonATHAN and PARK, 1995).

To provide an efficient polarization analysis method for surface wave arrivals, we implement in the time-domain a procedure based on the pure-state polarization method developed for frequency-domain analysis of highly polarized multidimensional waves by SAMSON and OLSON (1980). The method provides a direct and objective means for evaluating signal polarization characteristics, assuming that the signal is purely polarized (linearly or elliptically). In general, seismic signals are in a pure state only for limited durations and frequency bands; seismic ground motions are usually much more complex than a pure-state process and there are temporal variations of polarization state. Nevertheless, the pure-state representation provides a fast, simple, and intuitive means for identification of various seismic phases and for analysis of instantaneous polarization orientation. We find it to be particularly useful for rapid analysis of strongly dispersed surface wave signals, and document such applications in this paper. The time-domain representation of the pure-state signal allows us to resolve the polarization incoherence associated with the transition between different wave packets and to avoid a priori separation of the signal into windowed wave packets prior to analysis, as necessary for frequency-domain implementations. In addition, the calculation of various polarization parameters is straightforward, and yields basic results consistent with sophisticated techniques such as the principal-component or covariance matrix method (e.g., FLINN, 1965; Montalbetti and KanASEWich, 1970; SAMSON, 1983).

In the following we first describe the method drawing on the basic strategies of SAMSON and Olson (1980) and Vidale (1986), and then apply it to seismic data recorded in California to investigate the polarization characteristics of surface waves propagating along the circum-Pacific margin where strong lateral velocity gradients cause significant deviations from the great circle path. 


\section{Method}

We consider the three-component seismic time series at a single station, projected in the great-circle coordinate system with transverse component $u_{t}(t)$ (clockwise motion relative to the source is positive), radial component $u_{r}(t)$ (outward longitudinal motion from the source is positive), and vertical component $u_{z}(t)$ (upward is positive). The associated analytic signals (e.g., VIDALE, 1986) are given by:

$$
\begin{aligned}
& x_{1}(t)=u_{t}(t)+i \hat{u}_{t}(t), \\
& x_{2}(t)=u_{r}(t)+i \hat{u}_{r}(t), \\
& x_{3}(t)=u_{z}(t)+i \hat{u}_{z}(t),
\end{aligned}
$$

where $\hat{u}$ denotes Hilbert transform (PApoulis, 1984). We combine these complex displacement representations into the vector $\mathbf{x}(t)=\left[x_{1}(t), x_{2}(t), x_{3}(t)\right]^{T}$, where $T$ denotes transpose. By analogy to one-dimensional analytic signals, this vectorial signal may be written as

$$
\mathbf{x}(t)=\mathbf{R}(t) \exp (i \Phi(t)),
$$

where $\mathbf{R}(t)$ and $\Phi(t)$ are the instantaneous amplitude (slowly varying complex-valued vector) and phase (rapidly varying real-valued scalar), respectively. The length of $\mathbf{x}(t)$ is given by

$$
\|\mathbf{R}(t)\|=\left(Q_{t}^{2}+Q_{r}^{2}+Q_{z}^{2}\right)^{1 / 2}
$$

where

$$
Q_{t}=\left[u_{t}^{2}+\hat{u}_{t}^{2}\right]^{1 / 2}, \quad Q_{r}=\left[u_{r}^{2}+\hat{u}_{r}^{2}\right]^{1 / 2}, \quad Q_{z}=\left[u_{z}^{2}+\hat{u}_{z}^{2}\right]^{1 / 2}
$$

are instantaneous envelopes for the transverse, radial and vertical components of the signal, respectively. As an example, for a Rayleigh wave signal with the particle motion defined by the real vector $\mathbf{x}_{r}(t)=[0,-b \sin (\omega t), a \cos (\omega t)]^{T}$, where $\omega$ is the angular frequency and $a$ and $b$ are positive constants, the corresponding analytic signal is $\mathbf{x}(t)=\exp (i \omega t)[0, i b, a]^{T}$.

For most seismic wave analysis applications, we are interested in signals that are linearly or elliptically polarized. The particle motion due to the interference of multiple linearly-polarized oscillations is elliptically polarized, since the interference of an arbitrary number of linearly-polarized oscillations with the same frequency but different phase, amplitude, and direction of polarization is equivalent to two oscillations on mutually-perpendicular directions with a phase difference of $90^{\circ}$ (GAL'PERIN, 1984). We thus parallel SAMSON and Olson (1980) in constructing timedependent polarization state vectors that correspond to such 'pure' states.

By analogy to the spectral representation (SAMSON and OLSON, 1980), we call a process a pure state if 


$$
\mathbf{x}(t)=\rho(t) \mathbf{v}
$$

where $\mathbf{v}$ is a vector constant $\left(\mathbf{v}=\left[v_{1}, v_{2}, v_{3}\right]^{T}\right)$ in a unitary space, and $\rho(t)=|\rho(t)| \exp (i \sigma(t))$, a time-dependent scalar. In the following analysis we use $\xi$ and $\eta$ to denote the real and imaginary parts of $\mathbf{v}$, respectively. In (3) vector $\mathbf{v}$ is not uniquely defined, and in general its real and imaginary parts are not orthogonal $\left(\xi^{T} \eta \neq 0\right)$. To obtain a vector constant $(\mathbf{r})$ that does have orthogonal real $\left(\mathbf{r}_{1}\right)$ and and imaginary $\left(\mathbf{r}_{2}\right)$ parts $\left(\mathbf{r}=\mathbf{r}_{1}+i \mathbf{r}_{2}\right)$ (this provides the simplest representation of the pure state signal), we multiply vector $\mathbf{v}$ with a phase factor

$$
\mathbf{r}=\exp (-i \phi) \mathbf{v}
$$

It follows from (4)

$$
\begin{aligned}
& \mathbf{r}_{1}=\cos \phi \xi+\sin \phi \eta, \\
& \mathbf{r}_{2}=-\sin \phi \xi+\cos \phi \eta .
\end{aligned}
$$

If the phase shift, $\phi$, is taken as the solution of

$$
\exp (i 4 \phi)=\exp (i 4 \gamma)
$$

where

$$
\gamma=\frac{1}{2} \arctan \frac{2 \beta}{\alpha}, \quad \alpha=\xi^{T} \xi-\eta^{T} \eta, \quad \beta=\xi^{T} \eta
$$

then vectors $\mathbf{r}_{1}$ and $\mathbf{r}_{2}$ become orthogonal, and the pure-state process given by (4) can be written equivalently as $\mathbf{x}(t)=\rho^{\prime}(t) \mathbf{r}$, where $\rho^{\prime}(t)=\rho(t) \exp (i \phi)$. Note that equation (6) has four solutions

$$
\phi=m \frac{\pi}{2}+\gamma, \quad(m=0,1,2,3)
$$

with one corresponding to vector $\mathbf{r}=\mathbf{r}_{1}+i \mathbf{r}_{2}$ where $\mathbf{r}_{1}^{T} \mathbf{r}_{1} \geq \mathbf{r}_{2}^{T} \mathbf{r}_{2}$ and others $i \mathbf{r},-\mathbf{r}$, and $-i \mathbf{r}$. The sign of vector $\mathbf{r}$ is not uniquely defined, since both $\mathbf{r}$ and $-\mathbf{r}$ correspond to solutions of (6). With this in mind, vector $\mathbf{r}$ represents the direction of polarization of the process and hereinafter is referred to as the polarization-state vector.

By multiplying the polarization-state vector $(\mathbf{r})$ with a unitary rotation matrix $(Q)$ (e.g., Goldstein, 1980), we obtain

$$
\mathbf{r}^{\prime}=[0,0, a]^{T} \pm i[0, b, 0]^{T}=[0, \pm i b, a]^{T},
$$

where $\mathbf{r}^{\prime}=Q \mathbf{r}, a=\left(\mathbf{r}_{1}^{T} \mathbf{r}_{1}\right)^{1 / 2}, b=\left(\mathbf{r}_{2}^{T} \mathbf{r}_{2}\right)^{1 / 2}$, and $a \geq b$. Here $\mathbf{r}_{1}$ and $\mathbf{r}_{2}$ are parallel to the major and minor axes of the polarization ellipse and the ratio of the corresponding component amplitudes

$$
E=b / a
$$


is the ellipticity, as for the spectral representation developed by SAMSON and OLSON (1980). We define a third polarization-state vector orthogonal to the polarization plane as $\mathbf{r}_{3}$ given by the cross product of $\mathbf{r}_{1}$ and $\mathbf{r}_{2}\left(\mathbf{r}_{3}=\mathbf{r}_{1} \times \mathbf{r}_{2}=\xi \times \eta\right)$. We can use (5)-(9) to determine the orientation and geometry of the polarization ellipse of the time domain pure state signal, analogous to the spectral application of SAMSON and OLSON (1980), but now explicitly formulated for easy application to time domain signals.

For simplicity, we remove the sign ambiguity of the polarization-state vector (r) with the following procedure, in which we define the orientation of the polarizationstate vector and eliminate abrupt variations of calculated polarization parameters due to the ambiguity in specifying the polarization direction by $\mathbf{r}_{1}+i \mathbf{r}_{2}$ or $-\mathbf{r}_{1}-i \mathbf{r}_{2}$. For an assumed $\mathbf{r}=\mathbf{r}_{1}+i \mathbf{r}_{2}$, we write the largest, median, and smallest components of $\mathbf{r}_{1}$ as $\mathrm{A}, \mathrm{B}$, and $\mathrm{C}$ (i.e., $\mathrm{A} \geq \mathrm{B} \geq \mathrm{C}$ ), respectively. Then, we chose an integer, $n$, with

$$
n= \begin{cases}1 & \text { if } A=\max \left[\left|r_{11}\right|,\left|r_{12}\right|,\left|r_{13}\right|\right], \\ -1 & \text { if } A \neq \max \left[\left|r_{11}\right|,\left|r_{12}\right|,\left|r_{13}\right|\right],\end{cases}
$$

when $|A+C|>\mu(|A|+|C|)$; or

$$
n= \begin{cases}1 & \text { if }\left[\mathbf{r}_{1}-\mathbf{r}_{1}^{\prime}\right]^{T}\left[\mathbf{r}_{1}-\mathbf{r}_{1}^{\prime}\right] \leq\left[\mathbf{r}_{1}+\mathbf{r}_{1}^{\prime}\right]^{T}\left[\mathbf{r}_{1}+\mathbf{r}_{1}^{\prime}\right], \\ -1 & \text { if }\left[\mathbf{r}_{1}-\mathbf{r}_{1}^{\prime}\right]^{T}\left[\mathbf{r}_{1}-\mathbf{r}_{1}^{\prime}\right]>\left[\mathbf{r}_{1}+\mathbf{r}_{1}^{\prime}\right]^{T}\left[\mathbf{r}_{1}+\mathbf{r}_{1}^{\prime}\right],\end{cases}
$$

when $|A+C| \leq \mu(|A|+|C|)$. Here $\mu$ is a given, small positive number, and $\mathbf{r}_{1}^{\prime}$ in (10b) denotes the $\mathbf{r}_{1}$ vector obtained for the previous time step. Our final choice for the polarization-state vector is $\hat{\mathbf{r}}=n \mathbf{r}$ with $n$ being either 1 or -1 . This eliminates the ambiguity in distinguishing prograde motion from retrograde motion.

We use the polarization vectors to calculate various polarization parameters. Given two coordinate system, the Euler angles $(\theta, \phi, \psi)$ define the orientation of the new system $\left(x_{1}^{\prime}, x_{2}^{\prime}, x_{3}^{\prime}\right)$ relative to the original system $\left(x_{1}, x_{2}, x_{3}\right)$. If $x_{1}^{\prime}, x_{2}^{\prime}$, and $x_{3}^{\prime}$ are parallel to $\mathbf{r}_{1}, \mathbf{r}_{2}$, and $\mathbf{r}_{3}$, respectively, and $r_{i j}(i=1,2,3 ; j=1,2,3)$ denote the $j$-th component of $\mathbf{r}_{i}$ in the original system, then the Euler angles are given by

$$
\begin{aligned}
& \theta=\pi / 2 \\
& \phi=\arctan 2\left(r_{12}, r_{11}\right) \\
& \psi=\arctan 2\left(r_{13},\left(r_{11}^{2}+r_{12}^{2}\right)^{1 / 2}\right) .
\end{aligned}
$$

for a linearly polarized oscillation (i.e., $b=0$ ), and by

$$
\begin{aligned}
\theta & =\arccos \left[r_{33} /(a b)\right], \\
\phi & = \begin{cases}\arctan 2\left(r_{31},-r_{32}\right), & \left(\left|r_{33}\right| \neq a b\right), \\
\arctan 2\left(r_{12}, r_{11}\right), & \left(\left|r_{33}\right|=a b\right),\end{cases} \\
\psi & = \begin{cases}\arctan 2\left(r_{13} / a, r_{23} / b\right), & \left(\left|r_{33}\right| \neq a b\right), \\
0, & \left(\left|r_{33}\right|=a b\right),\end{cases}
\end{aligned}
$$


for an elliptically polarized oscillation $(b \neq 0)$, with $0 \leq \theta \leq \pi,-\pi \leq \phi<\pi$, and $-\pi \leq \psi<\pi$.

It is convenient to define geographic parameters for the azimuth and dip of the major polarization vector, $\mathbf{r}_{1}$. The azimuth is measured clockwise from north and given by

$$
\phi_{a}=\phi_{R}+2 m \pi+\arctan 2\left(r_{11}, r_{12}\right), \quad\left(-\pi \leq \phi_{a}<\pi\right),
$$

where $\phi_{R}$ is the azimuth of the radial direction, and $m$ an integer appropriate for $\phi_{a}$, ranging between $-\pi$ and $\pi$. The dip is given by

$$
\delta_{a}=-\arctan 2\left(r_{13},\left(r_{11}^{2}+r_{12}^{2}\right)^{1 / 2}\right), \quad\left(-\pi / 2 \leq \delta_{a} \leq \pi / 2\right) .
$$

We utilize these equations in our time-domain analysis to determine the instantaneous ellipticity and polarization orientation of seismic ground motions. The results obtained from this method are consistent with those for the time domain method of VIDALE (1986), in which the eigenvalue problem of a covariance matrix is explicitly solved and a numerical method is employed for searching for the direction of polarization. Prescribing the orientation of $\mathbf{r}_{1}$ using (10) removes the ambiguity in specifying the azimuth and dip of the polarization direction and the need for computation with various phase factors, as in the method of VIDALE (1986), simplifying the analysis for a time series. It is straightforward to show that for the correlation matrix $R$, given by

$$
R_{i j}=\frac{1}{M} \sum_{k=1}^{N} x_{i k} x_{j k}^{*}, \quad(i=1,2,3 ; j=1,2,3),
$$

where a set of $M$ observation with $x_{i k}=x_{i}\left(t_{k}\right),(k=1,2, \ldots, M)(M=3$ for threecomponent data) and the asterisk indicates complex conjugation, the eigenvectors of $R$ for a pure-state process are given by

$$
\begin{aligned}
& \mathbf{r}=\mathbf{r}_{1}+i \mathbf{r}_{2}, \\
& \mathbf{p}=\frac{\mathbf{r}_{1}}{a^{2}}-i \frac{\mathbf{r}_{2}}{b^{2}}, \\
& \mathbf{q}=\mathbf{r}_{3},
\end{aligned}
$$

and the direction of polarization is parallel to $\mathbf{r}$ (the polarization-state vector) and perpendicular to $\mathbf{p}$ and $\mathbf{q}$. Equation (15) indicates that the eigenvectors of the correlation matrix of a three-component signal are linear combinations of the polarization vectors of the signal. For a general process polarization characteristics are specified by the eigenvalues $\lambda_{i}$ and eigenvectors $\mathbf{u}_{i}(i=1,2,3)$ of the correlation matrix and expressions in SAMSON and Olson (1980), JEPSEN and KenNETT (1990) and Montalbetti and Kanasewich (1970) define the degree of rectilinearity and planarity. Estimation of uncertainties in frequency dependent polarization estimates based on singular value decomposition of the correlation matrix is discussed by PARK 
and Chave (1984) and Park et al. (1987). While the time domain formulation is not amenable to comparable error analysis, characterization of complex waveform behavior is often facilitated by treatment of the time series, which provided the motivation for our adaptation of the pure-state formulation to the time domain.

\section{Analysis of Surface Wave Data}

Our polarization analysis involves determination of the polarization vectors of the three-component time domain signals assuming pure state, and evaluating the orientation of the resultant vectors. In order to gain insight into the behavior of the polarization vectors, we show the time dependence of their three components, along with the measured orientation parameters. We demonstrate the polarization analysis procedure using synthetic and actual time series, and then define some procedures for measuring azimuths and instantaneous periods that are used in the subsequent data application.

\subsection{Application to Synthetic Time Series}

We consider transverse, radial, and vertical component time series consisting of two synthetic signals separated in time by $20 \mathrm{~s}$ (Figs. 1(a-c)). The first signal has a period of $10 \mathrm{~s}$ and is linearly polarized with the direction of polarization $\mathbf{r}_{1}=(-3,3.1,4.0)$ (where the components are given in the transverse, radial and vertical coordinates, respectively; the second signal has a period of $50 \mathrm{~s}$ and is elliptically polarized with the major and minor axes of the polarization ellipse $\mathbf{r}_{1}=(3.8,4,0)$ and $\mathbf{r}_{2}=(0,0,-3)$. The amplitudes at the beginning and termination of each signal are reduced by multiplying by half-sine functions.

To limit the analysis to signals with sufficiently large amplitudes for reliable determination of polarization parameters, we define a threshold, $\varepsilon$, for normalized instantaneous amplitudes of the analytic signal. The normalized instantaneous amplitudes are given by

$$
A_{k}=\left[a\left(t_{k}\right)^{2}+b\left(t_{k}\right)^{2}\right]^{1 / 2} / A M P, \quad(k=1, \ldots, N)
$$

with

$$
A M P=\max _{k}\left\{\left[a\left(t_{k}\right)^{2}+b\left(t_{k}\right)^{2}\right]^{1 / 2}\right\}
$$

the maximum of the instantaneous amplitudes for the signal observed at $N$ time steps. For time steps with normalized instantaneous amplitudes smaller than the threshold, the polarization parameters are reduced by multiplying them with a small factor. For example, the ellipticity is calculated by 


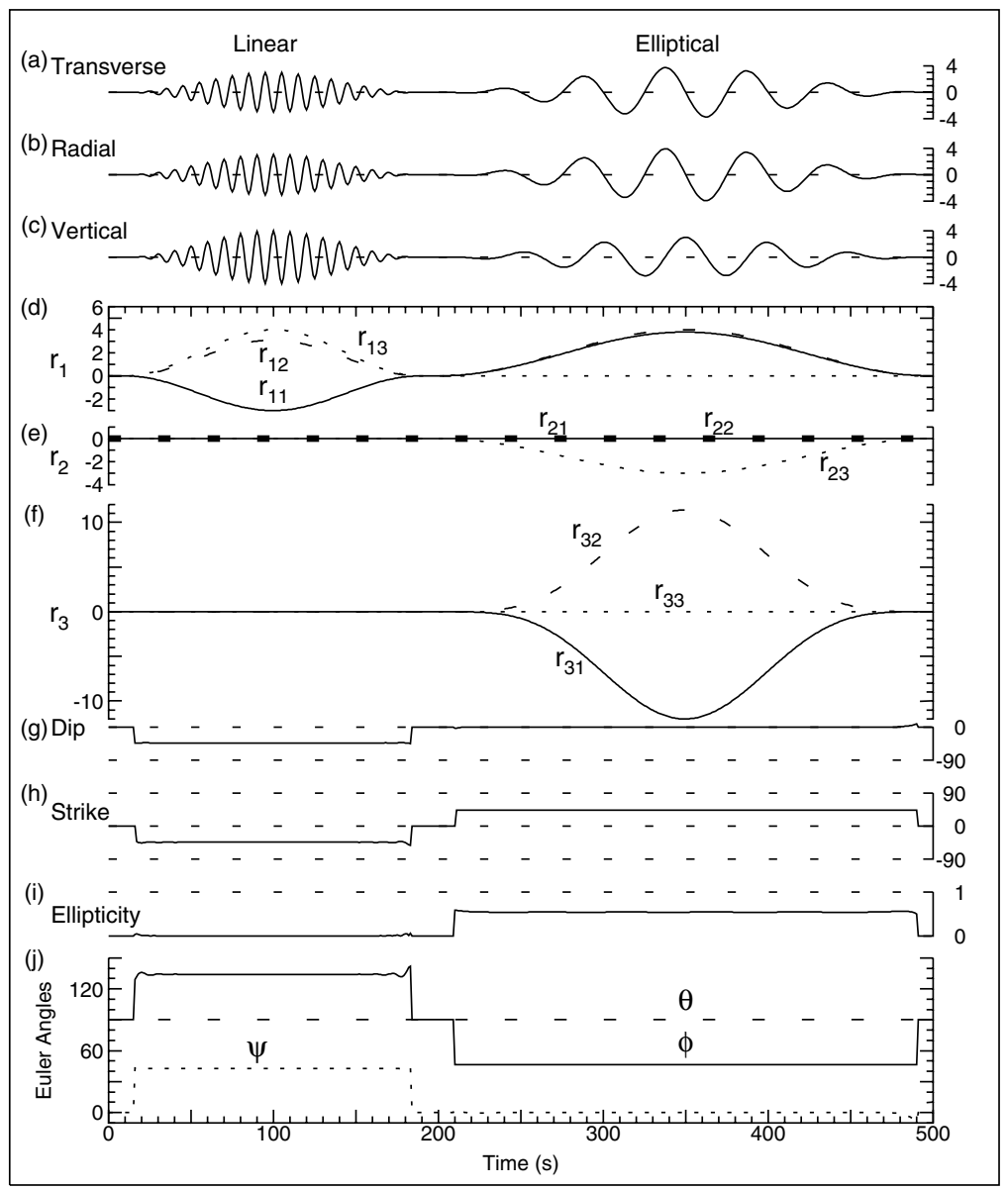

Figure 1

(a-c) The components of the synthetic time series. The first signal starts at about $10 \mathrm{~s}$ and is linearly polarized on a direction $-44^{\circ}$ in strike relative to the radial axis and $-43^{\circ}$ in dip; the second starts at about $20 \mathrm{~s}$ after termination of the first and is elliptically polarized with ellipticity $E=0.54$, and its major polarization vector $\mathbf{r}_{1}$ has a strike of $44^{\circ}$ and a dip of $0^{\circ}$. (d-f) The components of the polarization vectors for the signals (solid lines, transverse components; dashed, radial components; dotted, vertical components). For (a-e) the units are the same, but for (f) they are in squares of those for $(a-e)$. ( $g-j)$ Various parameters calculated using the polarization vectors: (g) dip and (h) strike or $\mathbf{r}_{1}$; (i) ellipticity; (j)

Euler angles $\theta, \phi$, and $\psi$. For dip, strike, and Euler angles, the units are in degrees.

$$
E\left(t_{k}\right)= \begin{cases}{\left[b\left(t_{k}\right) / a\left(t_{k}\right)\right] \exp \left[-\left(1-A_{k} / \varepsilon\right) \times 10^{5}\right],} & \text { if } \quad A_{k} \leq \varepsilon \\ b\left(t_{k}\right) / a\left(t_{k}\right), & \text { if } \quad A_{k}>\varepsilon .\end{cases}
$$

Other polarization parameters are calculated similarly. $\varepsilon$ is set to 0.01 .

Figures $1(\mathrm{~d}-\mathrm{f})$ show the polarization vectors which are obtained by assuming $\rho(t)=1$ in (3). The two signals are well isolated on various vector components, with 


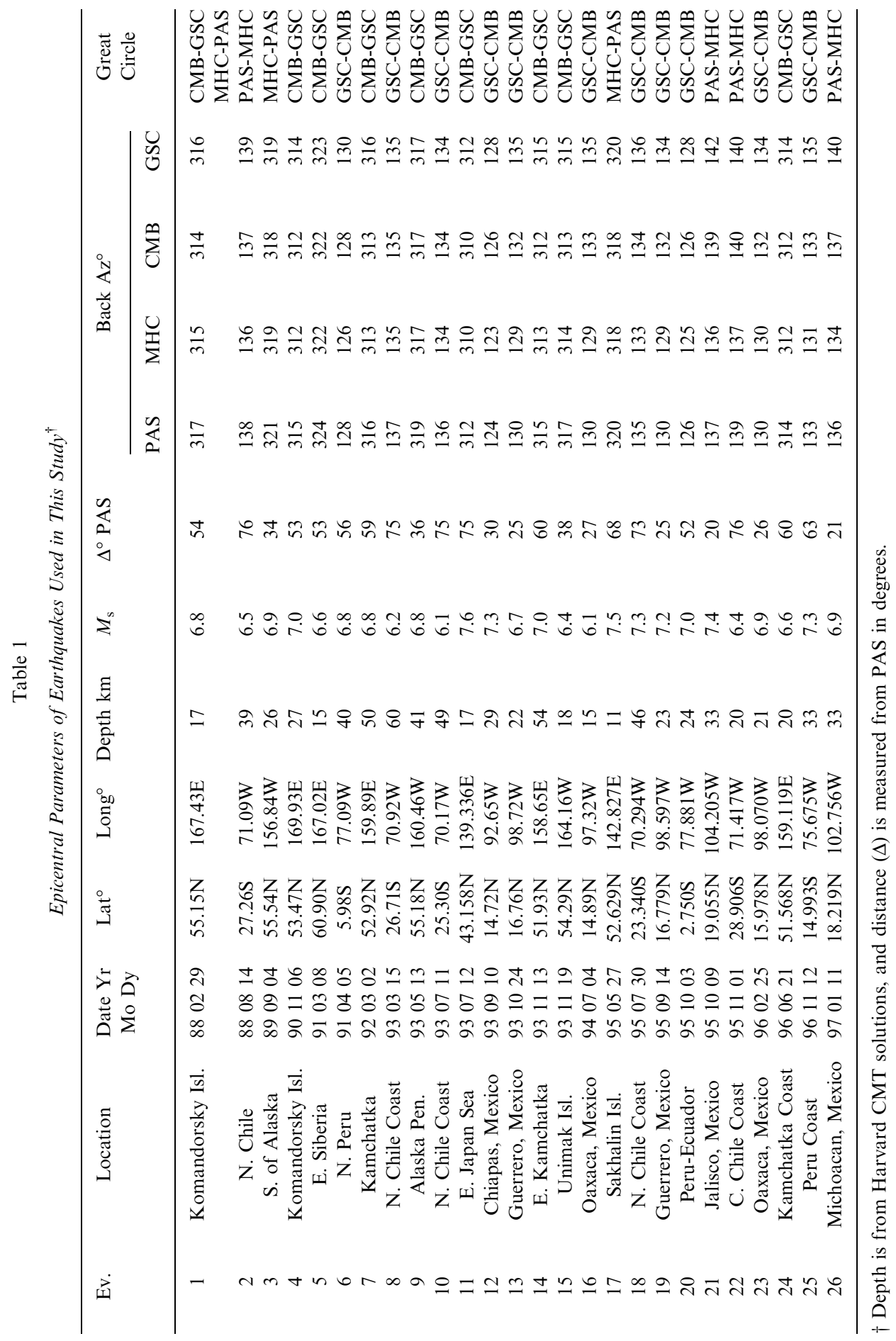


the linear signal isolated to $\mathbf{r}_{1}$ and the elliptical motion defined by not-zero values of $\mathbf{r}_{2}$. The polarization vector orthogonal to the polarization plane $\left(\mathbf{r}_{3}\right)$ is redundant, but helps to define the off great-circle deflection of the elliptical motion with large values of $r_{31}$ and $r_{32}$. Figures $1(\mathrm{~g}-\mathrm{j})$ show the dip, strike, and ellipticity of the polarization vectors, along with the redundant Euler angles, which are calculated using the purestate analysis method. The corresponding parameters for each synthetic signal are well predicted.

There are small erratic variations of the calculated polarization parameters near the beginning and termination of the signals (Figs. $1(\mathrm{~g}-\mathrm{j})$ ), as typically found by most methods. Using a threshold for normalized instantaneous amplitudes in the parameter determination, these variations are kept small. This test demonstrates that polarization vectors reliably extract key polarization information in the absence of noise. The performance degrades in the presence of noise and with overlapping signals, as the following data applications illustrate. Rather than conduct detailed error analysis on each spectral sample (a massive undertaking), we evaluate stability of results for multiple observations to assess the robustness of polarization estimates.

\subsection{Station and Event Distribution}

The data used are from earthquakes recorded by four broadband stations of the TERRASCOPE and BDSN (Berkeley Digital Seismic Network) networks: southern California stations PAS (Pasadena) and GSC (Goldstone) and central California stations MHC (Mt. Hamilton) and CMB (Columbia College). These stations span the active San Andreas Fault System (SAFS), and all events are near two great circle paths traversing California along the plate boundary. The MHC-PAS path is in the vicinity of the San Andreas Fault System (SAFS), with a length of $473 \mathrm{~km}$. The CMB-GSC path traverses of Sierra Nevada Batholith parallel to and east of SAFS, with a length of $441 \mathrm{~km}$.

Table 1 lists the epicentral parameters of the twenty-six earthquakes used in this study, which occurred between February, 1988 and January, 1997 with $M_{s} \geq 6.1$. The events were located toward the northwest or southeast from California within an arc distance of $90^{\circ}$ from the stations. Figure 2 shows the distribution of the stations and events along with great-circle paths that connect station PAS with the epicenters of three of the earthquakes. For every event the differences between back-azimuths for the four stations are smaller than $6^{\circ}$.

The earthquakes are thrust or strike-slip events. Figure 3 shows the corresponding radiation patterns of Rayleigh and Love waves at a period of $100 \mathrm{~s}$. For events 910405 (Peru), 930910 (Chiapas), 940704 (Oaxaca), 951009 (Jalisco), and 951101 (Chile), the stations are close to nodal azimuths of Rayleigh-wave radiation; for events 880814 (Chile) and 890904 (Alaska), which are shallow, low-angle thrust events, the stations are in the azimuths of strong Rayleigh but weak Love wave radiation; for events 880209 and 901106 (Komandorsky Islands), which are strike- 


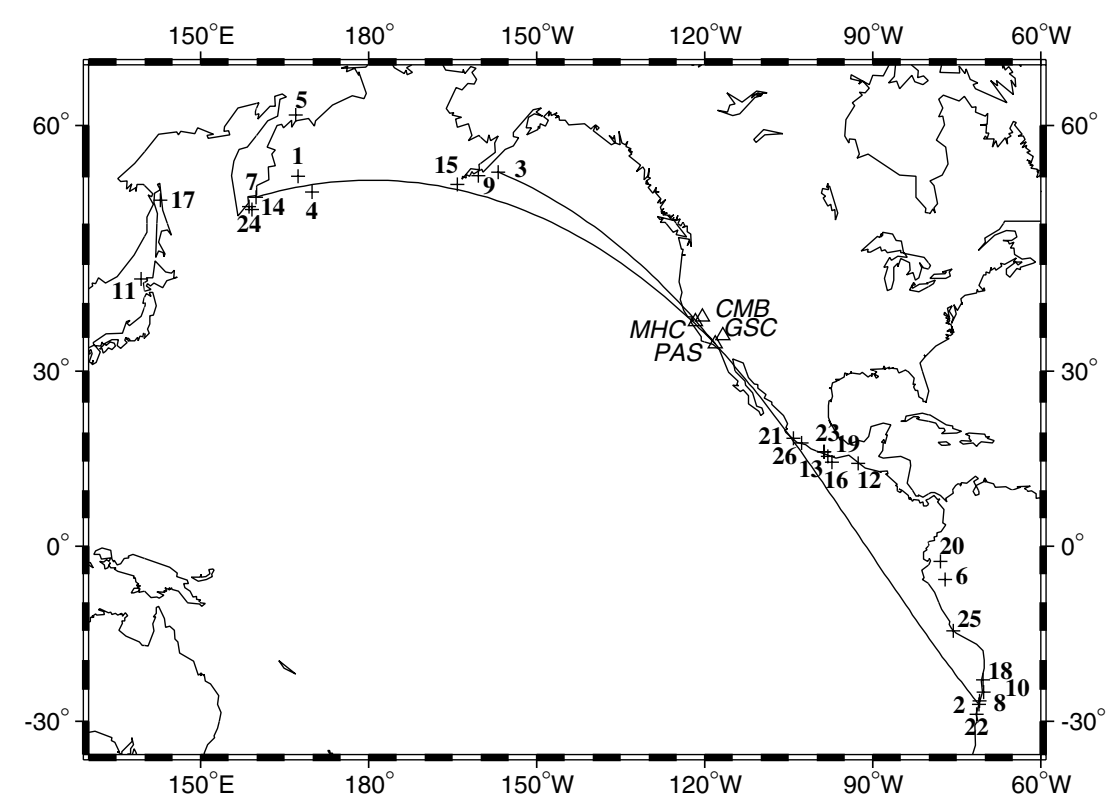

Figure 2

Location of stations (triangles) and earthquakes (plusses) used in this study. Source-receiver great-circle paths (solid lines) are shown for station PAS to three events. Earthquake epi-central parameters along with event numbers shown here are listed in Table 1.

slip events, the stations are in the azimuths of nodal Love wave radiation. This allows us to seek the arrival of anomalous wave packets such as quasi-Love $(Q L)$ or quasiRayleigh $(Q R)$ waves, which are generated by Love-to-Rayleigh or Rayleigh-to-Love conversion due to azimuthal anisotropy in the earth's upper mantle (YU and PARK, 1993).

\subsection{Polarization Vectors of Seismograms}

Polarization vectors and parameters were calculated for all seismograms, including various filtered traces, but we provide detailed results only for the broadband seismograms recorded at PAS from the 1989 Alaska earthquake (event 890904). Figure 4a shows the unfiltered seismograms. The $P, S$, Love (marked as $L Q$ ), and Rayleigh (marked as $L R$ ) waves are prominant, and relatively well separated. Figure $4 \mathrm{~b}$ shows the three components of each polarization vector, again relative to the transverse, radial and vertical coordinate system. Figure $4 \mathrm{~b}$ demonstrates that (1) $P$ waves are linearly polarized on the vertical and radial components with impulsive $r_{13}$, emergent $r_{12}$, and very weak other $r_{i j}$ components; (2) $S$ wave polarization is not purely linear and there is a small component on the minor vector $\left(\mathbf{r}_{2}\right)$ probably as a result of SPL energy; (3) Love waves are primarily 


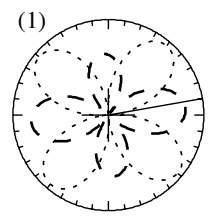

880229 Komand.

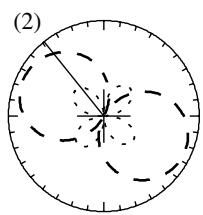

880814 N. Chile

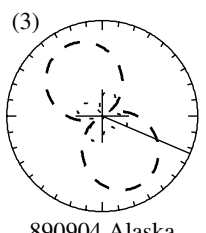

890904 Alaska

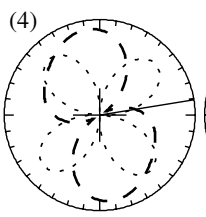

901106 Komand.

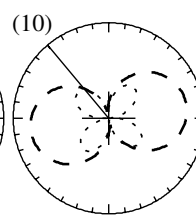

930711 N. Chile

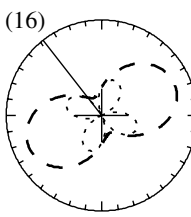

940704 Oaxaca

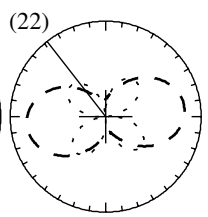

951101 C. Chile

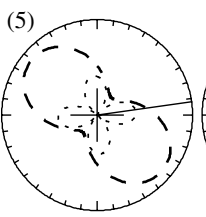

910308 E. Siberia

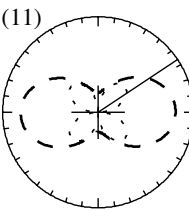

930712 Japan Sea
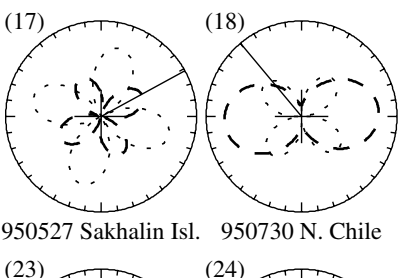

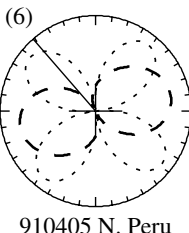

(12)
(13)

(19)

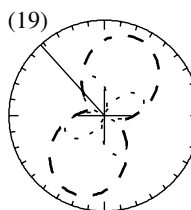

(20)

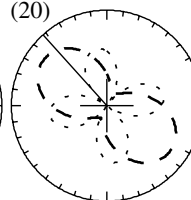

950914 Guerrero 951003 Peru-Ecuador
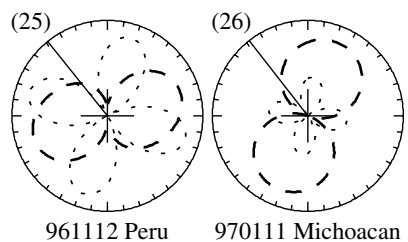

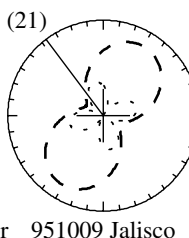

951009 Jalisco

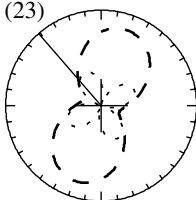

960225 Oaxaca

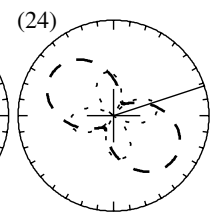

960621 Kamchatka

Figure 3

Amplitude radiation patterns or Rayleigh (dashed lines) and Love (dotted lines) waves at a period of $100 \mathrm{~s}$ for the earthquakes used in this study. For each earthquake the azimuth of station PAS is indicated by the solid line. The radiation patterns are computed for a point source with source parameters from the Harvard CMT solutions and for the average ocean model of REGAN and ANDERSON (1984).

linearly polarized on the transverse direction $\left(r_{11}\right)$, but there is some relatively shortperiod elliptical motion with nonzero $r_{22}$; (4) Rayleigh-wave arrivals are large and have substantial transverse $r_{11}$ and $r_{21}$ components. The small $r_{33}$ and large $r_{31}$ and $r_{32}$ indicate elliptical polarization on a vertical plane with significant off-great circle propagation.

In Figure $4 \mathrm{~b}$ symbols $R^{1}, R^{2}$, and $R^{3}$ in the Rayleigh wave window identify three somewhat discrete swings for $\mathbf{r}_{3}$ components, indicating time-varying polarization of Rayleigh wave arrivals with changing orientation of the vertical polarization plane. 


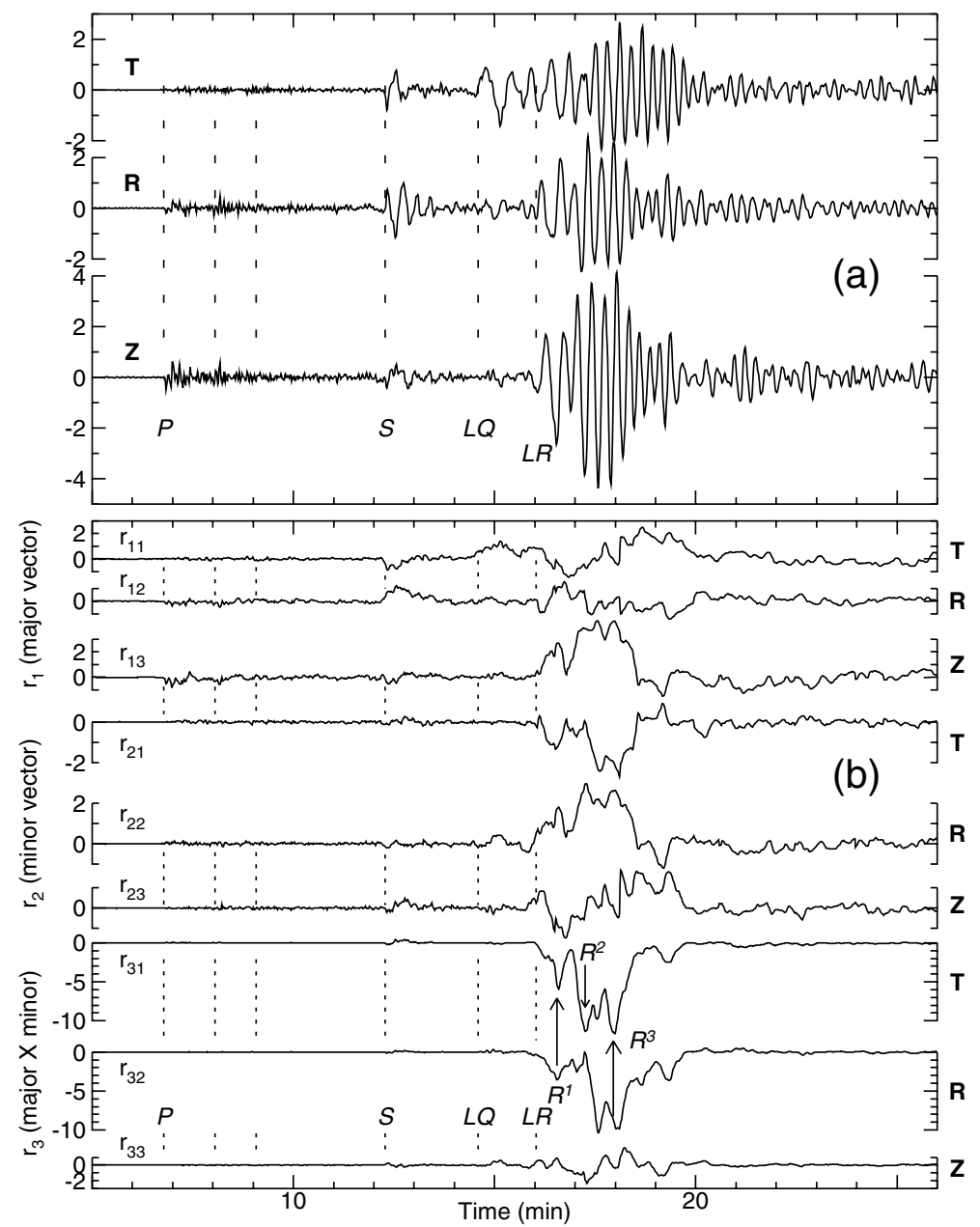

Figure 4

(a) Data (unfiltered GSN LH recordings) from the September 4, 1989 South of Alaska earthquake (event 890904, Table 1) recorded at station PAS (T, transverse; $\mathbf{R}$, radial; $\mathbf{Z}$, vertical), with units in $10^{5}$ counts for all three components. Time is relative to the origin time. The arrival times of $P, P P, P c P, S, L Q$, and $L R$ waves are from the NEIC and marked with dashed lines. (b) Various components of polarization vectors for the seismograms. The units are in $10^{5}$ counts for components of $\mathbf{r}_{1}\left(r_{11}, r_{12}, r_{13}\right)$ and $\mathbf{r}_{2}\left(r_{21}, r_{22}, r_{23}\right)$, and $10^{10}$ counts for $\mathbf{r}_{3}\left(r_{31}, r_{32}, r_{33}\right)$.

The $R^{1}$ signal early in the Rayleigh wave window has a moderate $r_{32}$ component, and may correspond to refracted Rayleigh waves or interference of refracted Rayleigh waves, scattered Love waves or $Q R$ waves. $Q R$ waves would appear on transverse components, after the Love wave but prior to the Rayleigh wave, and are best observed at long periods ( $T 70 \mathrm{~s}$ ) for shallow events near the Love source-radiation minima (PARK and YU, 1992, 1993; YU and PARK, 1993, 1994). However, when our transverse seismogram is low-pass filtered with a cut-off period of $70 \mathrm{~s}$ there is little 
energy following $L Q$; when high-pass filtered with a cut-off period of $100 \mathrm{~s}$, the transverse component arrivals are in phase with the $L R$ wave packet on the radial component, indicating refracted Rayleigh wave energy. In contrast with $R^{1}$, the following $R^{2}$ signal has very small $r_{32}$ and may correspond to direct Rayleigh waves propagating along the great circle path. The signals following $R^{2}$, in particular, the $R^{3}$ signal, have very large $r_{32}$ and again correspond to refracted Rayleigh waves.

Figures 5(a-c) show the envelope functions for the seismograms in Figure 4. For Love waves the instantaneous envelopes for radial and vertical components are

(a)

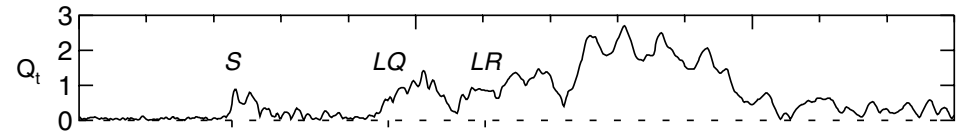

(b)

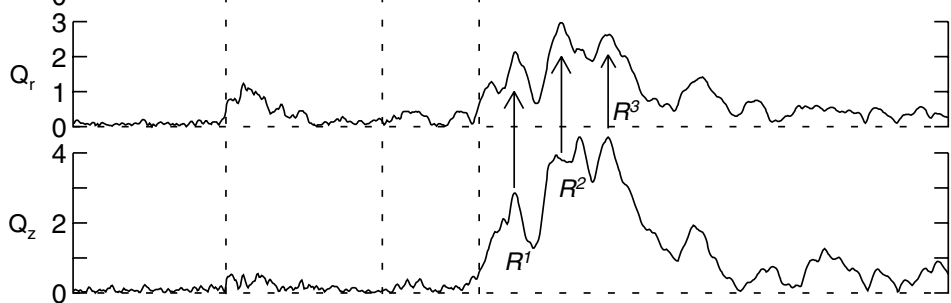

(d)

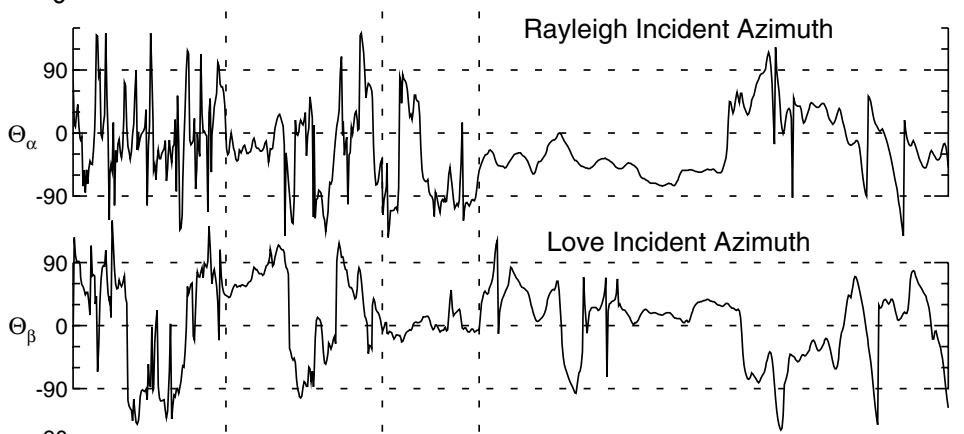

(f)

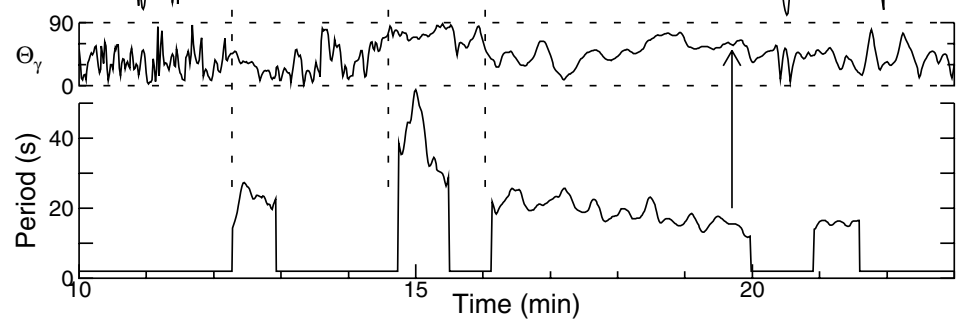

Figure 5

Variations of various polarization parameters for the seismograms shown in Figure 4. (a-c) Instantaneous envelopes (units in $10^{5}$ counts) of (a) transverse, (b) radial, and (c) vertical component seismograms; (d) azimuths of the intersection of the polarization plane and the horizontal plane relative to the radial direction; (e) azimuths of $\mathbf{r}_{1}$ relative to the transverse direction; (f) azimuths of the instantaneous envelope for horizontal components relative to the radial direction; (g) instantaneous periods. The arrow indicates the end of the time window for which the average azimuth of the Rayleigh-wave polarization plane is computed. 
much smaller than that for the transverse component, indicating linear polarization and near great-circle propagation; for Rayleigh waves the instantaneous envelope of the transverse component has amplitudes comparable with that of the radial component, but it spans a longer duration with large amplitudes than the other components.

\subsection{Incident Azimuths}

We now examine the incident azimuths, which are the azimuths of arrival at a given station (which may depart from the great-circle backazimuth). We use $\Theta_{\alpha}$ to denote the incident azimuth of Rayleigh waves (measured clockwise from the radial direction to the intersection of the polarization plane and horizontal plane), $\Theta_{\beta}$ the incident azimuth of Love waves (measured clockwise from the transverse direction to the direction of the major polarization vector $\mathbf{r}_{1}$ ), and $\Theta_{\gamma}$ the angle between the radial direction and the direction of vector-valued instantaneous envelopes for horizontal components. Figures $5(\mathrm{~d}-\mathrm{f})$ show variations of $\Theta_{\alpha}, \Theta_{\beta}$, and $\Theta_{\gamma}$, respectively, demonstrate the same characteristics of the Love and Rayleigh waves as discussed previously.

To determine the average incident azimuth for energy arriving over a given time window, we minimize the following function

$$
F=\sum_{k=1}^{N} \sin ^{2}\left(\bar{\zeta}-\zeta_{k}\right) W_{k}
$$

where subscript $k$ indicates the $k$-th time step $\left(t_{k}, k=1, \ldots, N\right)$ for the time window, $\zeta_{k}$ incident azimuths, $\bar{\zeta}$ the average of $\zeta_{k}$, and $W_{k}$ the weights, which represent the signalto-noise ratio (SNR). For Rayleigh waves $\zeta_{k}=\Theta_{\alpha}\left(t_{k}\right)$ and $\bar{\zeta}=\bar{\zeta}_{\alpha}$; for Love waves $\zeta_{k}=\Theta_{\beta}\left(t_{k}\right)$ and $\bar{\zeta}=\bar{\zeta}_{\beta}$. Using the weighted average incident azimuth $(\bar{\zeta})$ allows us to reduce contributions from signals with small amplitudes. For Love waves we use

$$
W_{k}=\frac{r_{11}\left(t_{k}\right)^{2}+r_{12}\left(t_{k}\right)^{2}}{A}\left(1-r_{13}\left(t_{k}\right)^{2} / B\right)
$$

with

$$
A=\max _{k}\left(r_{11}\left(t_{k}\right)^{2}+r_{12}\left(t_{k}\right)^{2}\right), \text { and } B=\max _{k}\left(r_{13}\left(t_{k}\right)^{2}\right) ;
$$

and for Rayleigh waves we use

$$
W_{k}=\frac{r_{31}\left(t_{k}\right)^{2}+r_{32}\left(t_{k}\right)^{2}}{A}\left(1-r_{33}\left(t_{k}\right)^{2} / B\right)
$$

with

$$
A=\max _{k}\left(r_{31}\left(t_{k}\right)^{2}+r_{32}\left(t_{k}\right)^{2}\right), \text { and } B=\max _{k}\left(r_{33}\left(t_{k}\right)^{2}\right) \text {. }
$$


By minimizing function (18a) we obtain the best estimate of $\bar{\zeta}$ and its error, which is the weighted RMS for $\bar{\zeta}-\zeta_{k}$.

For the long-period channel (LH) seismograms recorded at PAS for the 1989 Alaska earthquake, we obtained $\bar{\zeta}_{\beta}=-5^{\circ}\left( \pm 7^{\circ}\right)$ for a time window before the Rayleigh wave arrival, and $\bar{\zeta}_{\alpha}=-40^{\circ}\left( \pm 7^{\circ}\right)$ for a time window about four minutes long following the Rayleigh wave arrival. Measurements for all seismograms are discussed in the following sections.

\subsection{Instantaneous Periods}

In this section we examine instantaneous phase and its variations. Using the notation of (2) and (4), the instantaneous amplitude and phase are given by

$$
\mathbf{R}(t)=|\rho(t)| \mathbf{r},
$$

and

$$
\Phi(t)=\sigma(t)+\phi
$$

respectively. The time derivative of the instantaneous phase gives the instantaneous angular frequency

$$
\Omega(t)=\frac{d \Phi(t)}{d t} .
$$

The instantaneous period is $2 \pi / \Omega(t)$. For the pure-state process, $\Phi(t)$ and $\Omega(t)$ are given by

$$
\Phi(t)=m \frac{\pi}{2}+\gamma, \quad(m=0,1,2,3)
$$

and

$$
\Omega(t)=\frac{\alpha \beta^{\prime}-\alpha^{\prime} \beta}{\alpha^{2}+4 \beta^{2}},
$$

respectively, where prime indicates time derivative, and $\gamma, \alpha$, and $\beta$ are defined by (7).

Figure $5 \mathrm{~g}$ shows the variations of instantaneous periods for $S$, Love, and Rayleigh waves for the unfiltered seismograms shown in Figure 4a. For time-steps with $\alpha^{2}+\beta^{2}$ much smaller than its maximum for the entire time window, the instantaneous period is taken to be the period corresponding to the Nyquist rate.

\subsection{Polarization Vector of Ground Displacements}

We apply the polarization analysis to low-pass filtered ground displacement waveforms for various filter bands to estimate surface wave incident azimuths. We use low-pass filtered waveforms since analyses for bandpass filtered waveforms are subject to the bandwidth of the filter which must be adjusted according to the time and 
frequency resolution; in addition, bandpass filters usually generate stronger waveform distortions than low-pass filters for waveforms with significant amplitude variations, which make the onset of a signal difficult to observe (STEWART and Douglas, 1983).

Figure $6 \mathrm{a}$ illustrates the polarization vectors for low-pass filtered ground displacements with a cut-off period of 20 -s obtained from the seismograms shown
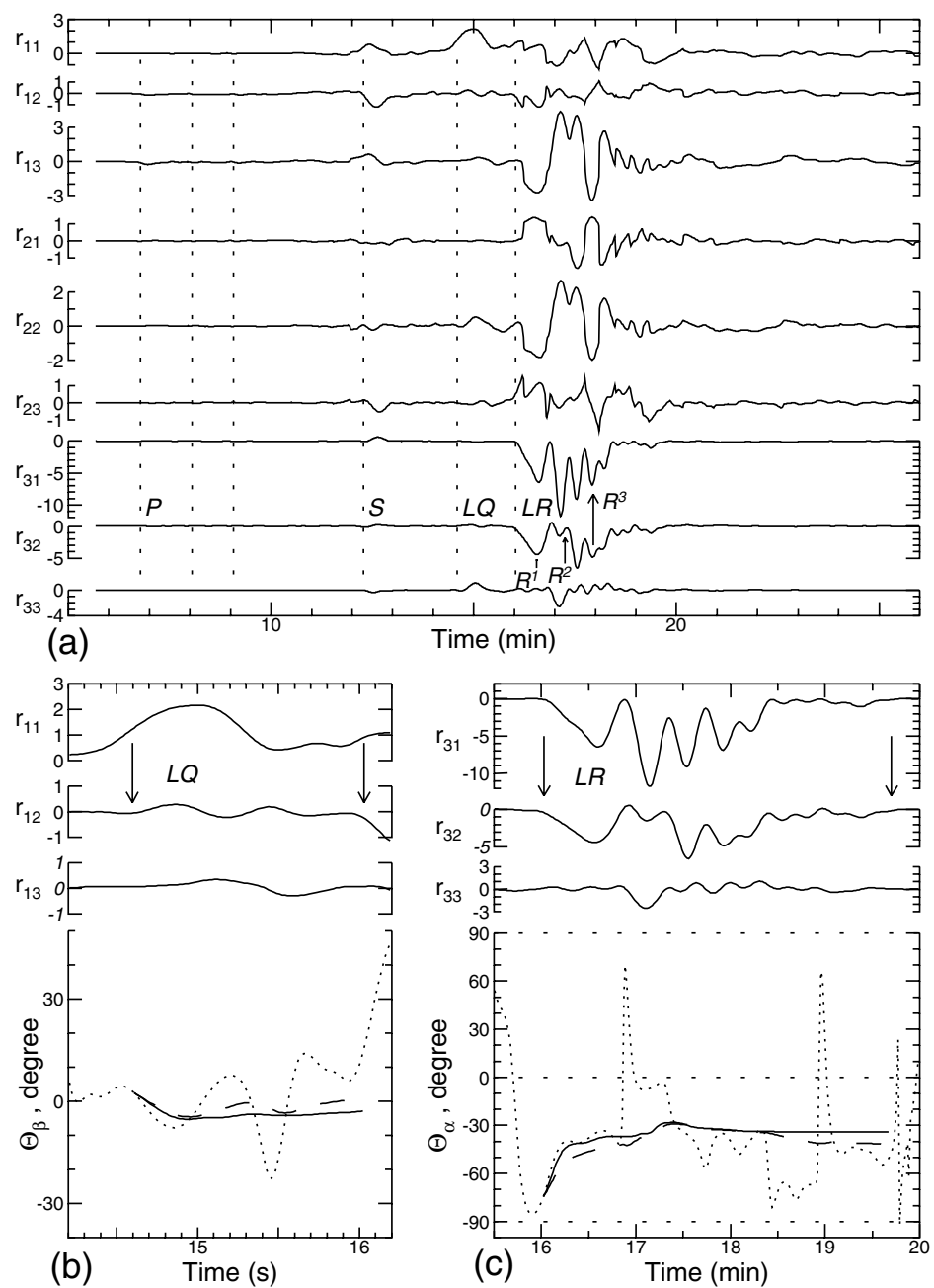

Figure 6

(a) The polarization vectors of ground displacements (filtered with a cut-off period of $20 \mathrm{~s}$ ) for the seismograms shown in Figure 4. The units are in $10^{2}$ microns for $\mathbf{r}_{1}$ and $\mathbf{r}_{2}$ and $10^{4}$ micron $^{2}$ for $\mathbf{r}_{3}$ components. (b) $\mathbf{r}_{1}$ components and Love wave incident azimuths (dotted line) relative to the transverse direction (solid line, weighted average of the azimuths; dashed line, unweighted average). (c) $\mathbf{r}_{3}$ components and Rayleigh wave incident azimuths. The left arrows in (b) and (c) indicate the Love and Rayleigh wave arrival times reported by the NEIC, the right arrows indicate the end of the time window for which the average incident azimuth is determined. 
in Figure 4a. The Rayleigh wave arrival corresponds to the abruptly increasing amplitudes of the vertical component of major vector $\mathbf{r}_{1}\left(r_{13}\right)$, and of all components of minor vector $\mathbf{r}_{2}$. The sharp amplification of $\mathbf{r}_{2}$ is characteristic of transition from linear polarization to elliptical or planar polarization. Figure $6 \mathrm{~b}$ shows the components of $\mathbf{r}_{1}$ along with measured incident azimuths $\left(\Theta_{\beta}\right)$ for Love waves. For time-steps that have small $r_{12}$ and $r_{13}$, the incident azimuths show large variations. The weighted mean for the incident azimuths, which is obtained using weight function (18b), shows smaller variations than the mean without weighting. For the Love wave time window shown in Figure 6b, the weighted mean is about $-3^{\circ} \pm 5^{\circ}$, while the mean without weighting is about $-2^{\circ}$, differing slighty from the weighted mean. Figure $6 \mathrm{c}$ shows the components of $\mathbf{r}_{3}$ along with measured incident azimuths $\left(\Theta_{\alpha}\right)$ for Rayleigh waves. The rapid variations of $\Theta_{\alpha}$ correspond to small $r_{31}$ and $r_{32}$. For various time windows beginning from the Rayleigh wave arrival, the mean incident azimuths that are obtained using variable weight coefficients (18c) show smaller variations than those obtained without weighting. With one-standard errors of about $6^{\circ}$, the weighted mean is about $-34^{\circ}$, while the mean obtained without weighting is about $-40^{\circ}$.

Figure 7a shows that for the same low-pass filtered seismograms, the instantaneous envelopes correlate well with $\alpha$ and $\beta$, and that the minima of $\alpha \beta^{\prime}-\alpha^{\prime} \beta$ and $\alpha^{2}+4 \beta^{2}$ correspond to small $\alpha$ and $\beta$, small instantaneous envelopes, and large variation of the instantaneous period. Therefore, to estimate the average instantaneous period, we use a weighted arithmetic mean with the following weights

$$
W_{k}=\left[\left(\alpha \beta^{\prime}-\alpha^{\prime} \beta\right)^{2}+\left(\alpha^{2}+4 \beta^{2}\right)^{2}\right] / A
$$

for the instantaneous period for the $k$-th time step, where $A$ is the maximum of $\left(\alpha \beta^{\prime}-\alpha^{\prime} \beta\right)^{2}+\left(\alpha^{2}+4 \beta^{2}\right)^{2}$ over the given time window. Using weight function (22), for displacement data filtered with a cut-off period of $20 \mathrm{~s}$ we obtained a mean instantaneous period of $30 \pm 2 \mathrm{~s}$ for Rayleigh waves and $55 \pm 5 \mathrm{~s}$ for Love waves. For the unfiltered seismograms shown in Figure 4a the average instantaneous period is about $23 \mathrm{~s}$ for Rayleigh waves and $39 \mathrm{~s}$ for Love waves. For a signal within a given time window we consider the average instantaneous period to be the dominant period of the signal.

The above procedure is applied to displacement data filtered with various cut-off periods. Figure $7 \mathrm{~b}$ shows polarization vectors for the same data filtered with a cut-off period of $100 \mathrm{~s}$. The weighted arithmetic mean for the instantaneous periods is $116 \pm 9 \mathrm{~s}$ for Rayleigh waves and $109 \pm 1 \mathrm{~s}$ for Love waves. The variations of the polarization vectors indicate that these long-period Rayleigh waves arrive approximately along the great-circle direction. The weighted average of incident azimuths relative to the greatcircle direction is $7^{\circ} \pm 2^{\circ}$ for Rayleigh waves and $10^{\circ} \pm 4^{\circ}$ for Love waves.

Figure $8 \mathrm{a}$ shows the measured incident azimuths as a function of the instantaneous period. The results indicate that for Rayleigh and Love waves with 

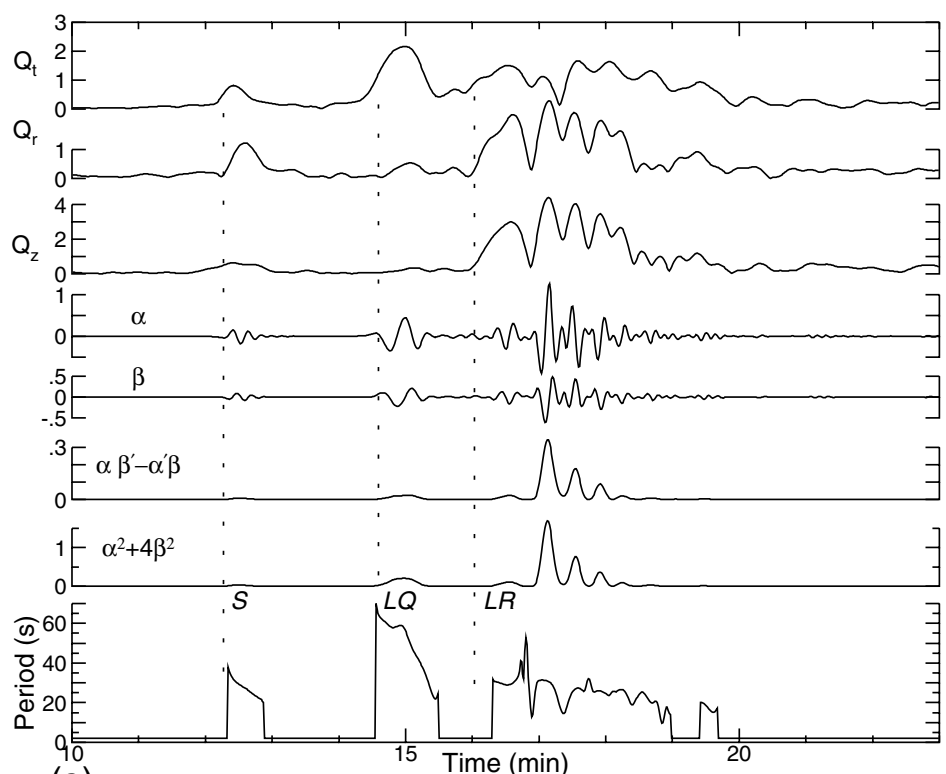

(a)
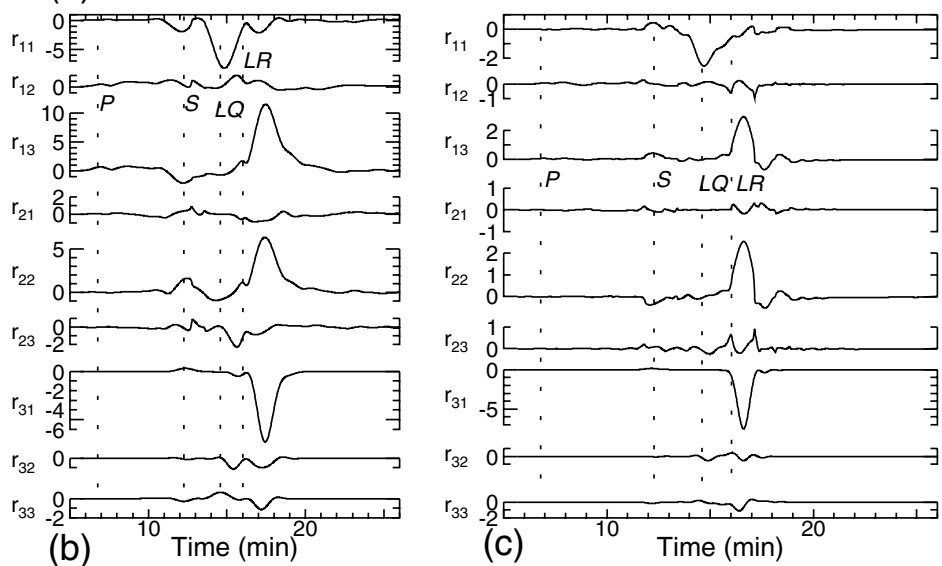

Figure 7

(a) The components of the instantaneous envelope of ground displacement for the seismograms shown in Figure 4 (filtered with a cut-off period of $20 \mathrm{~s}$ ), instantaneous-frequency parameters $\alpha, \beta, \alpha \beta^{\prime}-\alpha^{\prime} \beta$, $\alpha^{2}+4 \beta^{2}$, and instantaneous periods. Units are arbitrarily set. (b) The polarization vectors of displacements filtered with a cut-off period of $100 \mathrm{~s}$. (c) The polarization vectors of synthetic ground displacements filtered with the cut-off period of $50 \mathrm{~s}$, which are computed using the Harvard CMT solution and Earth model PREM. See Figure 6 for the units of various polarization vector components.

periods between 20 and $180 \mathrm{~s}$ the errors of the weighted mean incident azimuths are in general smaller than $10^{\circ}$; for Love waves with periods between 50 and $150 \mathrm{~s}$ and for Rayleigh waves with periods between 70 and $150 \mathrm{~s}$, the errors are smaller than $5^{\circ}$; and for Rayleigh and Love waves with periods longer than $180 \mathrm{~s}$ the incident 
(a) 1989 South of Alaska (Event 890904)
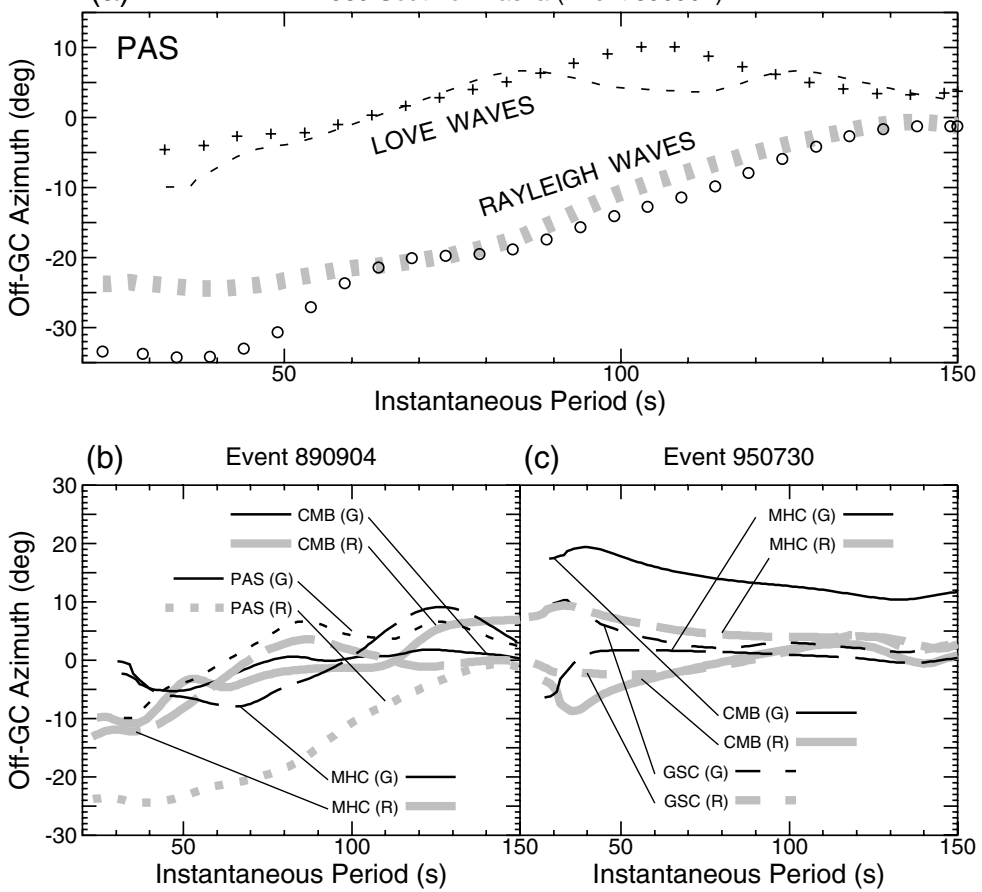

Figure 8

(a) The incident azimuths versus instantaneous period, which are obtained using polarization vectors for ground displacements of surface waves recorded at PAS from the 1989 Alaska earthquake. Results obtained without corrections for mode contamination are shown in open circles (Rayleigh waves) and crosses (Love waves); results obtained with the corrections are shown in shaded line (Rayleigh waves) and dashed line (Love waves). (b, c) The incident azimuths of surface waves recorded at various stations from (b) the 1989 Alaska earthquake (event 890904) and (c) the 1995 N. Chile coast earthquake (event 950730).

azimuths are smaller than $5^{\circ}$, although the errors become much larger due to small SNR. Finally, for periods shorter than $30 \mathrm{~s}$ the incident azimuths of Love waves can not be determined well from the data used in this study. The strong azimuthal deflections for Rayleigh waves resolved in Figure 8 are robust, and consistent with results obtained by other polarization analysis procedures.

\subsection{Correction for Mode Contamination}

In the procedure described above for calculation of the incident azimuths we assume that mode contamination or interference between waves with different polarization characteristics is small and can be ignored. To better take into account mode contamination, we applied the above procedure to synthetic seismograms computed for anisotropic Earth model PREM for all modes with periods longer than 32 s using the method of Gilbert and Dziewonski (1975). Figure 7c shows the 
polarization vectors for the synthetic displacement seismograms filtered with a cutoff period of $50 \mathrm{~s}$ for station PAS and the 1989 South of Alaska earthquake. The overlap of Love and Rayleigh wave energy is evident on the horizontal and vertical components of vectors $\mathbf{r}_{1}$ and $\mathbf{r}_{2}$, and this can contaminate azimuth measurements.

Guided by the synthetic results the measured incident azimuths are corrected for mode contamination using relative amplitudes of various components of polarization vectors for synthetic seismograms. The corrections are made using vector $\mathbf{r}_{3}$ for Rayleigh waves and $\mathbf{r}_{1}$ and $\mathbf{r}_{2}$ for Love waves. Figure $8 \mathrm{a}$ shows the incident azimuths corrected for mode contamination along with the azimuths obtained without the correction, indicating that the corrections for periods longer than $80 \mathrm{~s}$ are much smaller than for periods between 30 and $60 \mathrm{~s}$.

\subsection{Path Dependent Incident Azimuths of Surface Waves}

For the data set used in this study, we observed strong incident-azimuth anomalies for Rayleigh waves with periods between 20 and $150 \mathrm{~s}$, with the anomalies for many paths being larger than $35^{\circ}$ for a period of $30 \mathrm{~s}$ and $20^{\circ}$ for a period of 150 s. For Love waves the anomalies are usually smaller than for Rayleigh waves.

Figure $8 \mathrm{~b}$ shows the measured incident-azimuth anomalies of surface waves as a function of period for the 1989 South of Alaska earthquake. The earthquake has good SNR for Rayleigh and Love waves and a large Rayleigh-Love amplitude ratio in comparison with other events used in this study (Fig. 3). For Love waves the anomalies are in general less than $5^{\circ}$ and do not show significant variations among stations; however, for Rayleigh waves with periods less than $100 \mathrm{~s}$ the anomalies at PAS are greater than $10^{\circ}$, differing significantly from those for MHC and CMB.

Figures $9(\mathrm{a}-\mathrm{c})$ show seismograms bandpass filtered between 30 and $200 \mathrm{~s}$ for six earthquakes, for which large Love or Rayleigh wave anomalies are found. For the waveforms recorded at PAS for the 1989 Alaska earthquake (Fig. 9a, top right panel), within the Rayleigh wave time window there is an anomalous arrival on the transverse component, which is in phase with the $R_{1}$ radial component. This anomalous arrival results in the large incident-azimuth anomalies of Rayleigh waves shown in Figure 8b.

Significant polarization anomalies of Rayleigh waves are also found for several other events. Figure $9 \mathrm{~b}$ shows examples for two of the earthquakes. For the waveforms with large polarization anomalies of Rayleigh waves, the transverse component of the anomalous arrival for the Rayleigh wave time window is usually in phase with the radial component of the Rayleigh wave packet. This arrival has about the same group velocity as $R_{1}$ and becomes stronger as waves propagate towards the southeast for events in the northwest (e.g., Figs. 9a and 9b, top panels) and toward the northwest for events in the southeast (e.g., Fig. 9b, bottom panels). We attribute the anomalous arrival to the refraction of Rayleigh waves rather than Rayleigh-toLove conversion or $Q R$ waves. In addition to the refraction, energy of scattered Love 

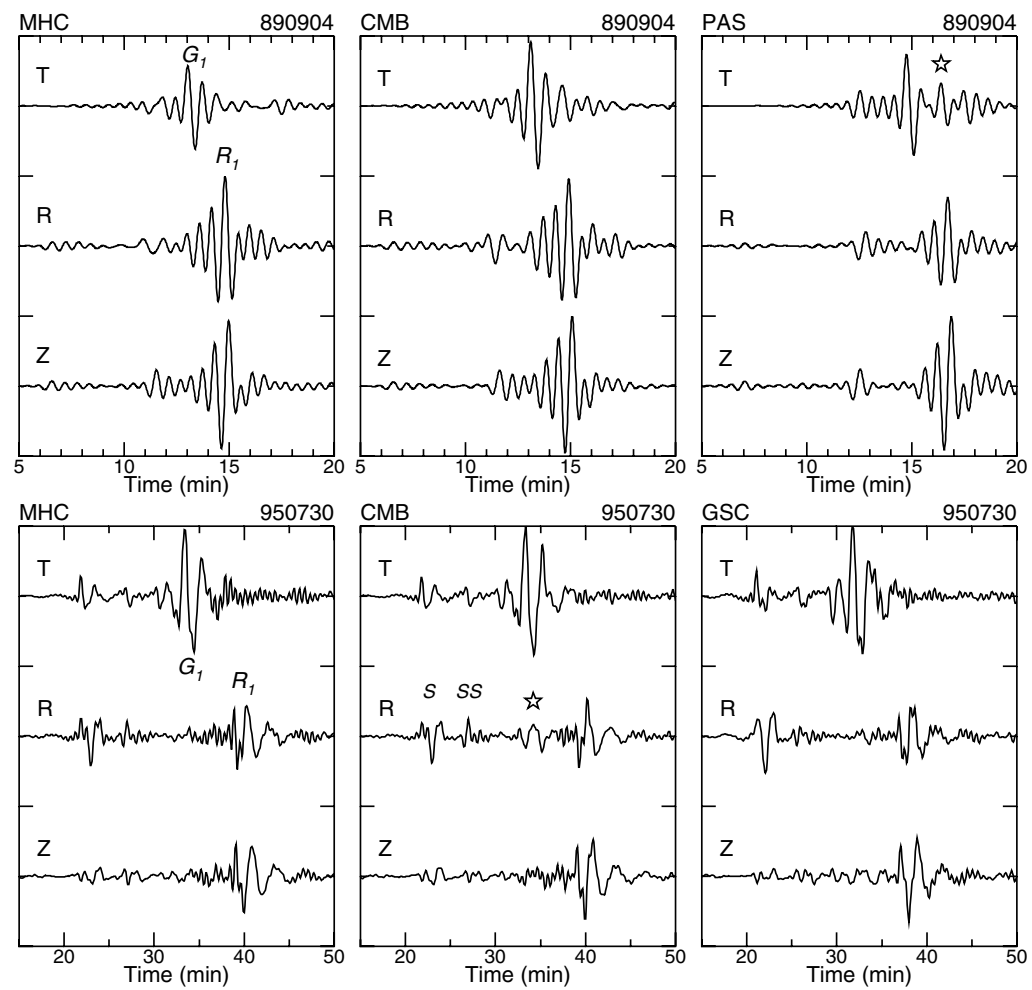

Figure 9a

Seismograms from the 4 September 1989 South of Alaska (event 890904, top panels) and 30 July 1995 near coast of Northern Chile (event 950730, bottom panels) earthquakes bandpass filtered between 30 and $200 \mathrm{~s}$. The stars in the PAS-890904 and CMB-950730 panels mark the refracted Rayleigh and Love wave anomalies, respectively.

waves may also contribute to the transverse component within the Rayleigh wave window.

Some waveforms exhibit large Love wave polarization anomalies. In comparison with the incident-azimuth anomalies for the 1989 South of Alaska event (Fig. 8b), the anomalies for the 1995 near coast of Northern Chile earthquake show significantly different variations as a function of period (Fig. 8c). The waveforms for the earthquake are shown in the bottom panels of Fig. 9a. For station CMB there is an anomalous arrival on the radial component for the Love wave time window, which corresponds to the large incident-azimuth anomalies of Love waves shown in Figure $8 \mathrm{c}$. The arrival has about the same group velocity as $G_{1}$ waves and a relatively small vertical component, and its radial component is in phase with Love waves on the transverse component.

For other events that show significant polarization anomalies of Love waves, the anomaly also corresponds to an anomalous arrival on the radial component for 

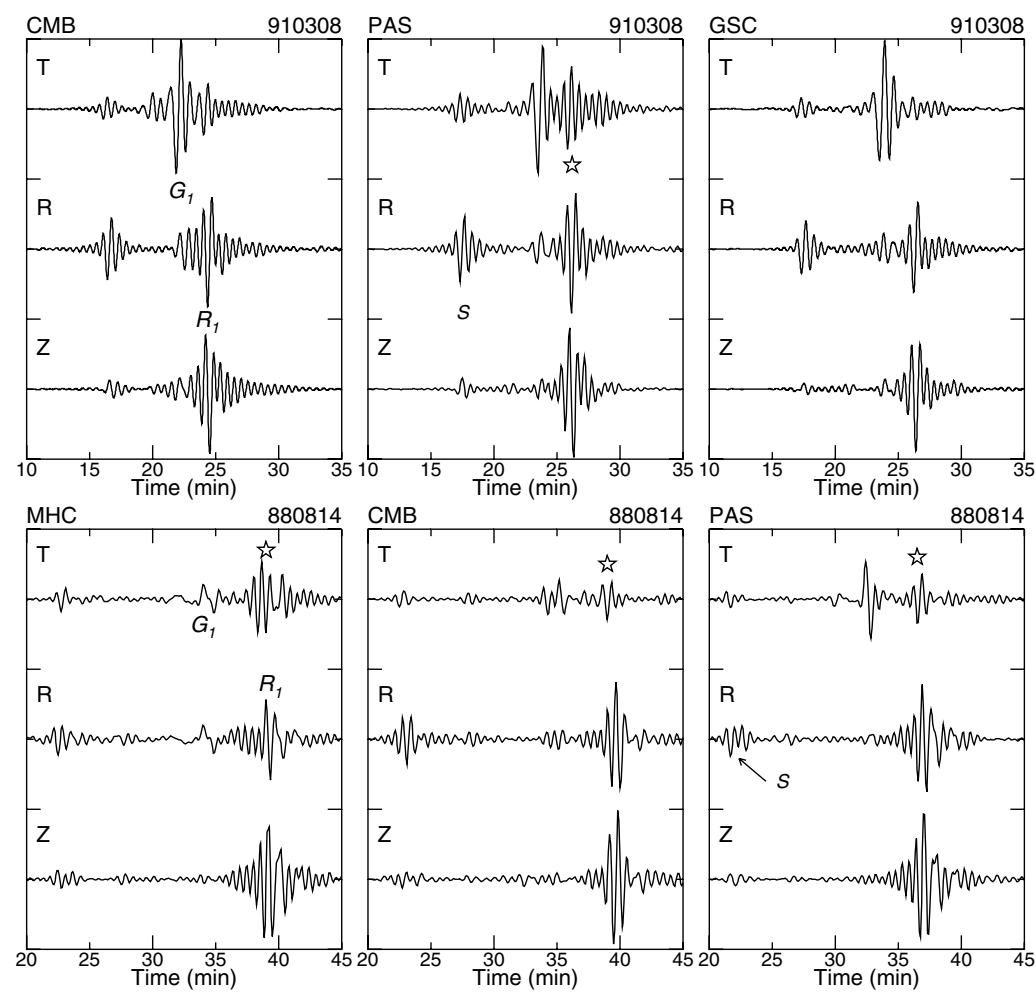

Figure $9 b$

Seismic data from the 8 March 1991 Eastern Siberia (event 910308, top panels) and 14 August 1988 near northern Chile coast (event 880814 , bottom panels) earthquakes. The stars mark the refracted Rayleigh wave anomalies.

the Love wave time window. Figure 9c shows the seismic data for two such events. In the waveforms from these events, there are usually large $S$ phases along with noticeable $S S$; and the Love waves usually overlap or follow the SSS phase. The $S$ phase usually has large radial or transverse and small vertical components, and is linearly or elliptically polarized; and the $S S$ usually shows polarization with larger ellipticity than $S$. For the data recorded at CMB from the 1992 Kamchatka earthquake (Fig. 9c, top left panel), both the radial and vertical components of the anomalous arrival are in phase with the transverse component of $G_{1}$ wave packet. For the data recorded at CMB from the 1996 Peru earthquake (Fig. 9c, bottom middle panel), the radial component of the anomalous arrival is in phase with the transverse component but out of phase with the vertical component. For both events, the vertical component of the arrival is smaller than the radial component, and the amplitude ratio of the radial and vertical components remains about the same for data low-pass filtered at $50 \mathrm{~s}$. Therefore, we attribute the anomalous arrival on the radial component for the Love wave time window to the combined effects of the 

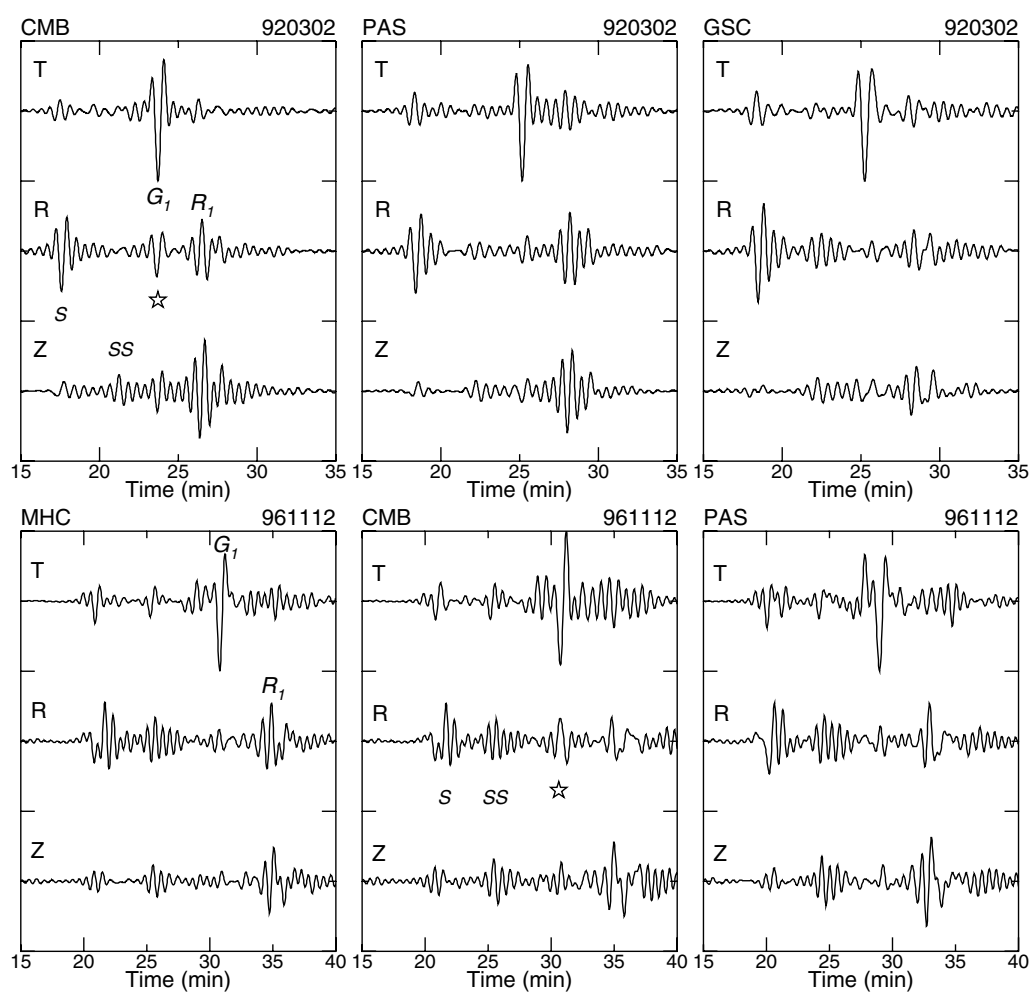

Figure 9c

Seismic data from the 2 March 1992 off east coast of Kamchatka (event 920302, top panels) and 12 November 1996 near coast of Peru (event 961112, bottom panels) earthquakes. The stars in the CMB920302 and CMB-961112 panels mark the anomalous phases on the radial component.

refracted Love waves and multiple or scattered $S$ waves. While for many events the Love wave anomalies recorded at $\mathrm{CMB}$ are larger than those for other stations, for several events in the northwest the anomalies for CMB are smaller than for PAS and GSC.

Figures 10 and 11 show the measured incident-azimuth anomalies for all the events used in this study. For several events listed in Table 1, records for some stations have small signal-to-noise ratio or glitches or are not available due to station operations. The data set has more records for station CMB than for other stations, while it includes only a few records for station MHC.

For the earthquakes located to the northwest from the stations, the variations of the anomalies as a function of period show similarities between various events (Figs. 10 and 11). However for the earthquakes in the southeast, the anomalies show somewhat stronger path dependency and variations as a function of period, indicating stronger laterally heterogeneities below paths from the southeast than for 


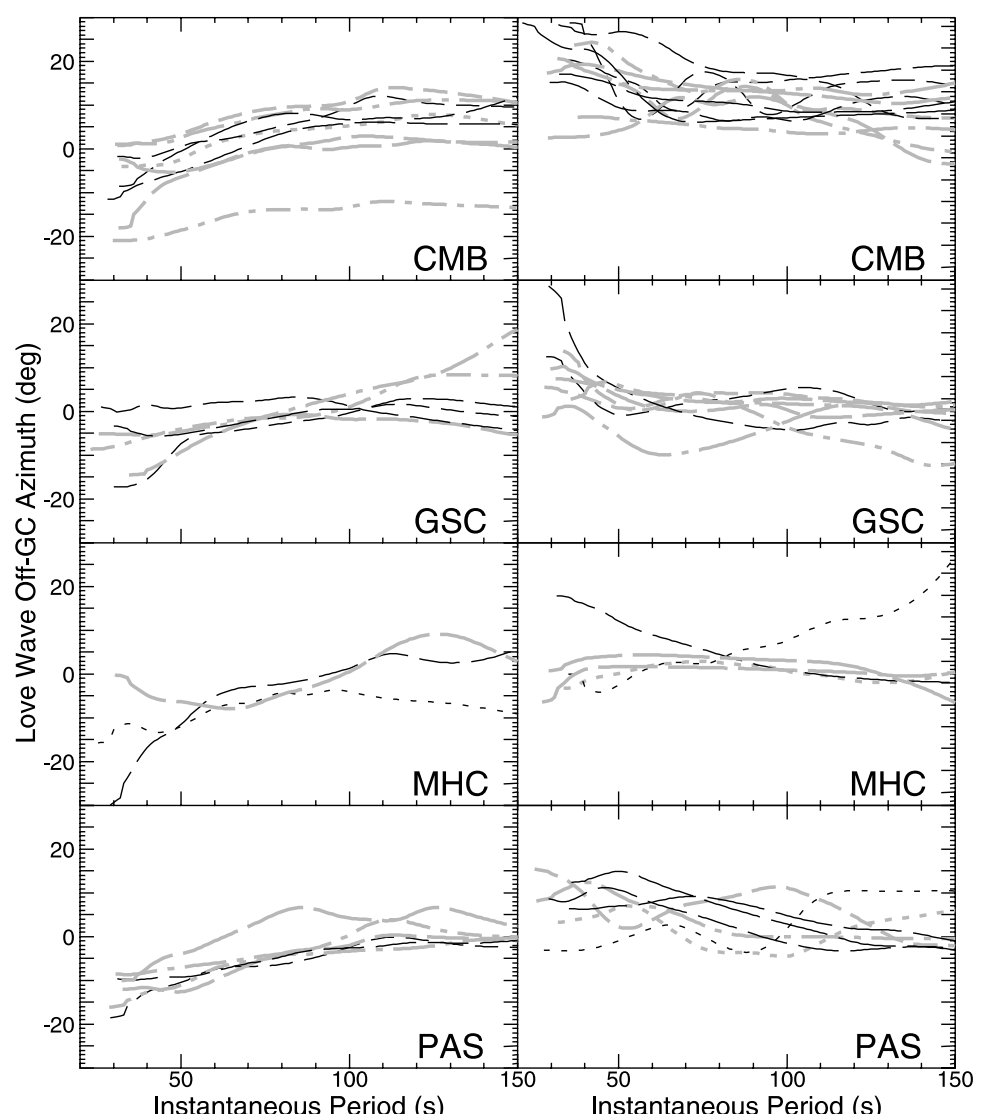

Northwest event $\Delta^{0}$ Northwest event $\Delta^{0}$ Southeast event $\Delta^{0}$ Southeast event $\Delta^{0}$

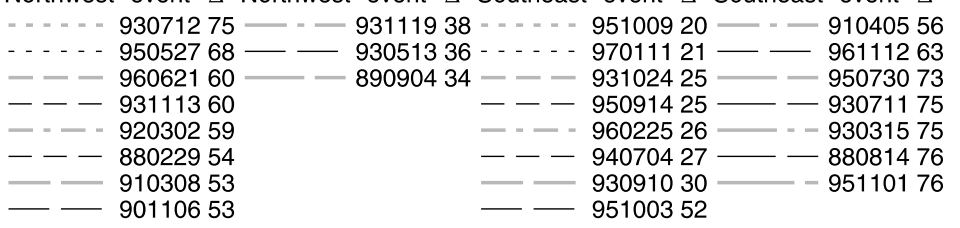

Figure 10

Incident-azimuth anomalies versus instantaneous period for Love waves recorded at various stations from the earthquakes located northwest (left panel) and southeast (right panel) of the stations.

northwest. As refractions may occur anywhere along the paths, some variability between events at the same azimuths is to be expected. Therefore, a large number of records must be collected to obtain a reliable average of the surface wave propagation anomalies. This is true for all polarization methods.

Figures $12(\mathrm{a}-\mathrm{c})$ show the directions of arrivals of surface waves with period of 30 s along with several source-station paths. For each station the azimuth of an arrow indicates the observed incident-azimuth anomaly. Figures $12 \mathrm{a}$ and $12 \mathrm{~b}$ show the 


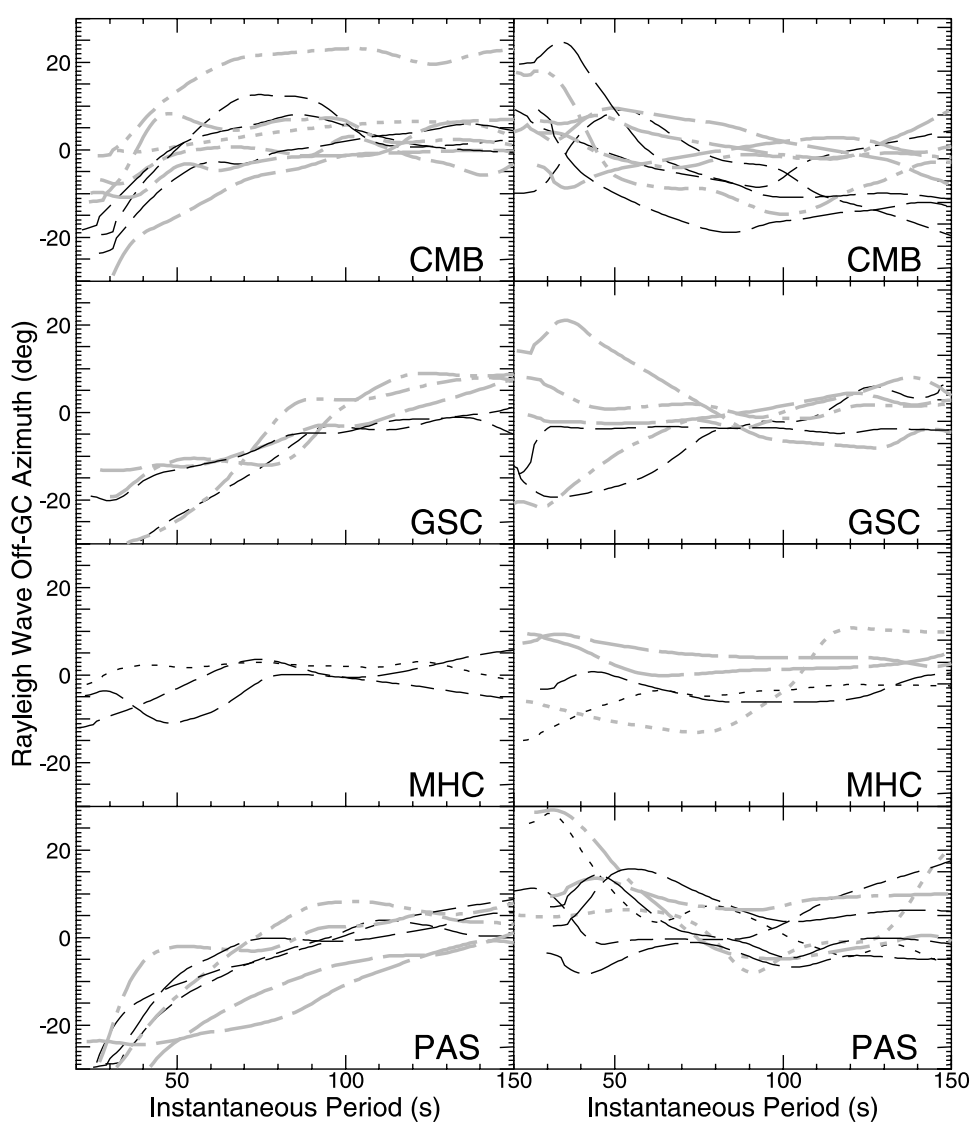

Northwest event $\Delta^{0}$ Northwest event $\Delta^{0}$ Southeast event $\Delta^{0}$ Southeast event $\Delta^{0}$

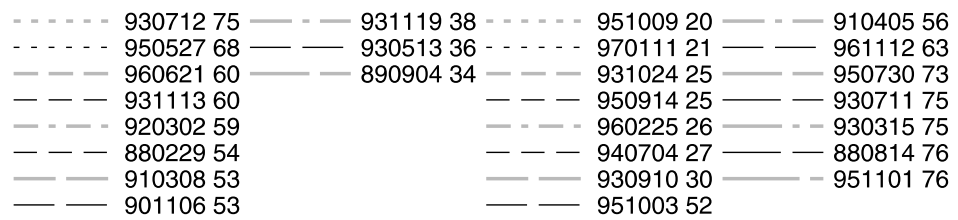

Figure 11

Incident-azimuth anomalies versus instantaneous period for Rayleigh waves recorded at various stations.

average directions of arrivals for all the events. For the earthquakes to the northwest (Fig. 12a), Rayleigh waves arrive at the stations with ray paths bending eastward about $10^{\circ}$ at $\mathrm{CMB}$ and $\mathrm{MHC}$ and about $25^{\circ}$ at PAS and GSC, indicating that the incident-azimuth anomalies become larger as waves travel to the south from $\mathrm{MHC}$ to PAS and from CMB to GSC. Figure 12c shows the arrival directions of Rayleigh waves from two earthquakes that have the best signal-to-noise ratio for the events in the northwest. The arrival directions are consistent between these events for PAS and MHC. 


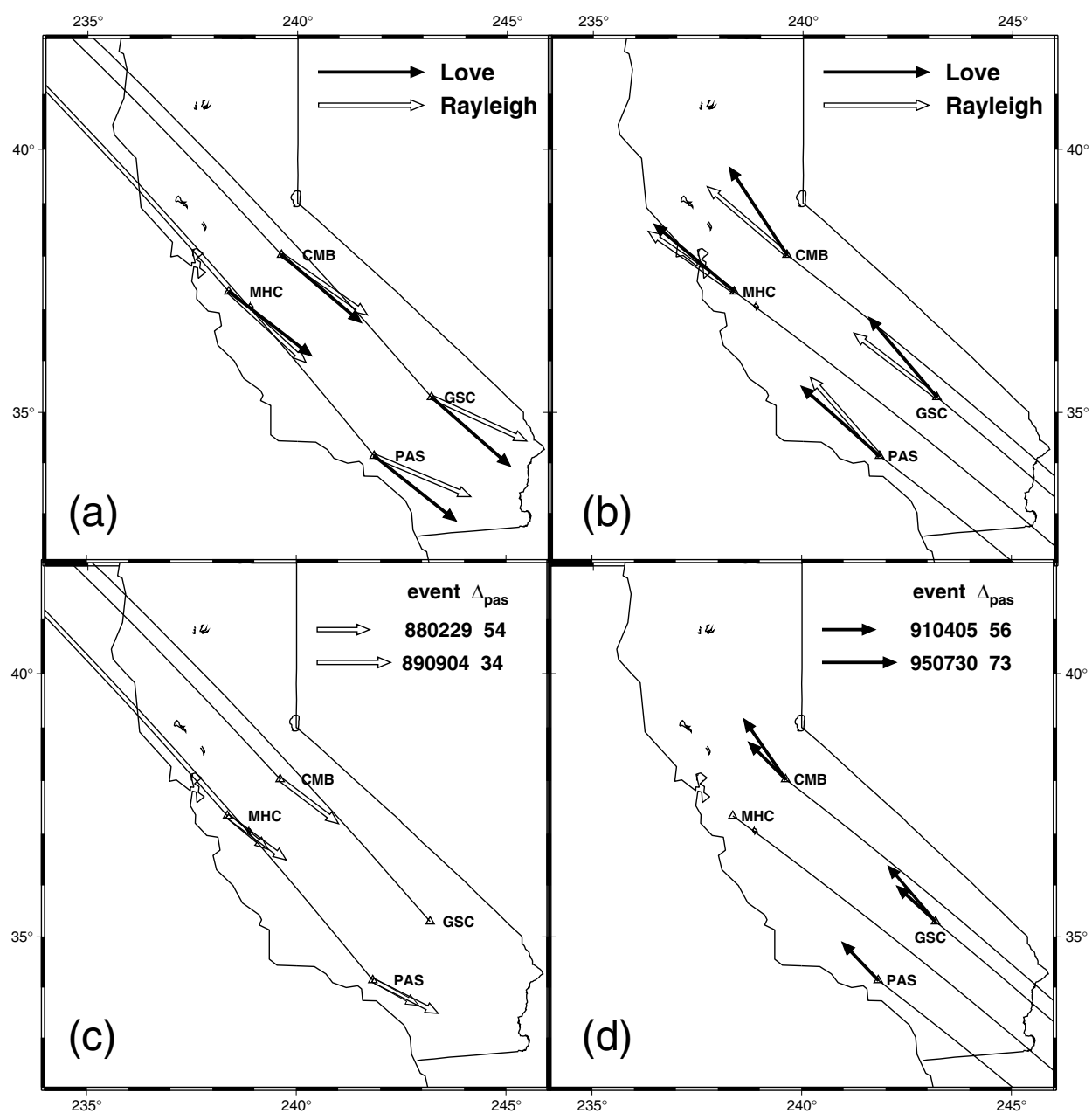

Figure 12

Directions of arrivals for surface waves with a period of $30 \mathrm{~s}$ (open arrows, Rayleigh waves; solid arrows, Love waves). (a) and (b) show average arrival-directions for the earthquakes located in the NW and SE, respectively. (c) and (d) show arrival-directions for selected events, for which the data have high SNR. The solid lines indicate great circle paths for the 1989 South of Alaska earthquake (event 890904). For each station the azimuth of an arrow relative to the solid line indicates observed incident-azimuth anomaly.

For Love waves, Figure 12a shows that the ray paths bend eastward about $10^{\circ}$ at all the stations, and there is no significant change of the direction as waves travel to the south. The difference between the results for Rayleigh and Love waves may reflect the differences between the effects of lateral heterogeneities on Rayleigh and Love waves and the intrinsic depth dependence of the various wavetypes. 
The data from earthquakes to the southeast show a more complex pattern (Fig. 12b). Some of the complexity may be due to low signal amplitudes in the data or due to strong effects of lateral heterogeneities near the sources. Figure $12 \mathrm{~d}$ shows the arrival directions of Love waves from two earthquakes, which have the best signalto-noise ratio for the events in the southeast. The Love waves arrive at GSC with ray paths bending eastward about $5^{\circ}$ and at $\mathrm{CMB}$ about $10^{\circ}$.

\section{Discussion}

Previous work has found strong path deflections for short to intermediate period surface waves. The magnitudes of the surface wave polarization anomalies found in this study are comparable to those found, for example, on Central Eurasia paths analyzed by LEVSHIN et al. (1992), but substantially larger than for fundamental mode surface waves (below $40 \mathrm{mHz}$ ) across the northwest Pacific (LERNER-LAM and PARK, 1989). There can be large differences between surface wave anomalies for paths along oceanic, continental, and tectonic plate boundary structures. The large anomalies found in California preclude application of two-station methods for measuring dispersion or attenuation, and motivate the use of array data to track wavefront deflections.

The observed incident-azimuth anomalies may be attributed to the large-scale lateral heterogeneities of structures below the wave paths. For the events in the northwest the differences between the anomalies for various stations suggest that wave velocities to the southwest of the paths are faster than those to the northeast. For some events, such as event 890904, the anomalies are observed for periods as long as $100 \mathrm{~s}$, suggesting that the lateral velocity gradient to the southwest of the paths may extend to deep depths, rather than being localized in the shallow crust.

In order to constrain the structure, we need to combine analyses of polarization and travel-time tomography. A successful example in this area is given by LASKE and MASTERs (1996), in which an aspherical earth model is developed using surface-wave phase, amplitude, and polarization data. ZHANG (1998) shows that the model obtained by LASKE and MASTERS (1996) provides improved global resolution in determination of earthquake source parameters. Recent work has begun to map out the strong lateral gradient in lithospheric thickness beneath California, with a westward increase in thickness by $50 \mathrm{~km}$ (MELBOURNE and Helmberger, 2001). This is likely to cause some of the strong azimuthal deflections that we observe. Three-dimensional modeling procedures and extensive polarization analysis of the California broadband network data should contribute to resolving this structure in future efforts. Network measurements will be needed to resolve the extent to which lateral gradients in structure beneath California is 
responsible for the polarization anomalies versus gradients elsewhere along the great-circle paths.

YU and PARK (1994) observed strong $Q L$ waves on many propagation paths in the Pacific Ocean region and attributed the observations to the existence of strong lateral gradients in azimuthal anisotropy in the region. The $Q L$ waves are elliptically polarized on the vertical and radial components and arrive slightly behind the Love wave but prior to the Rayleigh wave (YU and PARK, 1994). For out data set, $Q L$ waves are not clearly observed, suggesting the absence of strong effects of anisotropy for the paths used in this study. This may result from differences between directions of the paths and the anisotropy axes or from the absence of strong lateral gradients in azimuthal anisotropy in the circum-Pacific region.

\section{Conclusion}

This study presents a time-domain procedure for analysis of polarization characteristics of three-component seismic waves building on the pure-state approach of SAMSON and OLSON (1980). Three orthogonal polarization vectors are calculated analytically from the three-component signal, and associated polarization measures for the time series are extracted from the vectors. Calculation of the vectors involves a total of nine components, but the procedure is straightforward and provides an intuitive basis for evaluating varying polarization in the time series. The method is applied to data recorded at several broadband stations in California from twenty-six large, shallow earthquakes that occurred between 1988 and 1997 . We investigate the polarization characteristics of surface waves propagating along the northern and eastern circum-Pacific. The earthquakes are located toward the northwest and southeast along the Pacific coast on the great circle paths connecting either stations MHC and PAS or stations CMB and GSC. The first path (MHCPAS) is in the vicinity of the San Andreas Fault System (SAFS), and the second (CMB-GSC) traverses the Sierra Nevada Batholith, parallel and to the east of the SAFS. Our analyses indicate that Rayleigh waves are strongly refracted, with ray paths bending eastward as waves travel toward the south, while Love waves are less affected. Strong lateral gradients in lithospheric structure are probably responsible, and future inversion for structure can utilize polarization measurements like those made in this study. Our data set shows little evidence of quasi-Rayleigh and quasiLove waves, indicating the absence of strong effects of azimuthal anisotropy for the paths considered.

\section{Acknowledgments}

The data used in this study were made available courtesy of BDSN/UC Berkeley, TERRAscope/Caltech, and DMC/IRIS network personnel. We thank the 
anonymous reviewers for constructive criticism that improved the presentation. This work was supported by AFOSR grant F49620-94-1-0315 and IGPP-LLNL grants B263983 and B283796 with facilities support from the W. M. Keck Foundation. Additional support from grants AFOSR F49620-95-1-0028 (to RuShan Wu) and NSF EAR 9418643 (to Thorne Lay). Contribution number 225 of the Institute of Tectonics and W. M. Keck Seismological Laboratory, University of California, Santa Cruz.

\section{REFERENCES}

Archambeau, C., Bradford, J., Broome, P., Dean, W., Flinn, E., and Sax, R. (1965), Data Processing Techniques for the Detection and Interpretation of Teleseismic Signals, Proc. IEEE 53, 18761884.

Aster, R. C., Shearer, P. M., and Berger, J. (1990), Quantitative Measurements of Shear Wave Polarizations at the Anza Seismic Network, Southern California: Implications for Shear Wave Splitting and Earthquake Prediction, J. Geophys. Res. 95, 12,449-12,473.

Aster, R. C., Shearer, P. M., and Berger, J. (1991), Reply, J. Geophys. Res. 96, 6415-6419.

Booth, D. C., Crampin, S., Lovell, J. H., and Chiu, J.-M. (1991), Temporal Changes in Shear-wave Splitting during an Earthquake Swarm in Arkansas, J. Geophys. Res. 95, 11,151-11,164.

Bungum, H. and CAPOn, J. (1974), Coda Pattern and Multipath Propagation of Rayleigh Waves at NORSAR, Phys. Earth and Planet. Int. 9, 111-127.

CAPon, J. (1970), Analysis of Rayleigh Wave Multipath Propagation at LAS, Bull. Seismol. Soc. Am. 60, 1701-1731.

Crampin, S. (1970), The Dispersion of Surface Waves in Multilayered Anisotropic Media, Geophys. J. R. astr. Soc. 21, 387-402.

Crampin, S. (1975), Distinctive Particle Motion of Surface Waves as a Diagnostic of Anisotropic Layering, Geophys. J. R. astr. Soc. 40, 177-186.

Crampin, S., Booth, D. C., Evans, R., Peacock, S., and Fletcher, J. B. (1990), Changes in Shear-wave Splitting at Anza near the Time of the North Palm Springs Earthquake, J. Geophys. Res. 95, 11,19711,212 .

Crampin, S., Booth, D. C., Evans, R., Peacock, S., and Fletcher, J. B. (1991), Comment on "Quantitative Measurements of Shear-wave Polarizations at the Anza Seismic Network, Southern California: Implications for Shear-wave Splitting and Earthquake Prediction" by Richard C. Aster, Peter M. Shearer, and Jon Berger, J. Geophys. Res. 96, 6403-6414.

FLInN, E. A. (1965), Signal Analysis Using Rectilinearity and Direction of Particle Motion, Proc. IEEE 53, 1874-1876.

Gal'Perin, E. I., The Polarization Method of Seismic Exploration (D. Reidel, Boston. 1984).

Gilbert, F. and Dziewonski, A. M. (1975), An Application of Normal Mode Theory to the Retrieval of Structure Parameters and Source Mechanisms from Seismic Spectra, Phil. Trans. R. Soc. Lond. A. 278, 187-269.

Goldstein, H., Classical Mechanics, 2nd edition (Addison-Wesley, Reading. 1980).

Jepsen, D. C. and Kennett, B. L. N. (1990), Three-component Analysis of Regional Seismograms, Bull. Seismol. Soc. Am. 80, 2032-2052.

Jobert, N. and Jobert, G. (1983), An Application of Ray Theory to the Propagation of Waves along a Laterally Heterogeneous Spherical Surface, Geophys. Res. Lett. 10, 1148-1151.

Jonathan, M. L. and Park, J. (1995), Multiwavelet Spectral and Polarization Analyses of Seismic Records, Geophys. J. Int. 122, 1001-1021.

Kanasewich, E. R., Time Sequence Analysis in Geophysics, 3rd edition (University of Alberta Press, Edmonton, Canada. 1981). 
Kirkwood, S. C. and Crampin, S. (1981), Surface Wave Propagation in an Ocean Basin with an Anisotropic Upper Mantle: Observations of Polarization Anomalies, Geophys. J. R. astr. Soc. 64, 487497.

Laske, G. and Masters, G. (1996), Constraints on Global Phase Velocity Maps from Long-period Polarization Data, J. Geophys. Res. 101, 16,059-16,076.

LAy, T. and KanAmori, H. (1985), Geometric Effects of Global Lateral Heterogeneity on Long-period Surface Wave Propagation, J. Geophys. Res. 90, 605-621.

Lerner-Lam, A. L. and PARK, J. (1989). Frequency-dependent Refraction and Multipathing of 10-100 Second Surface Waves in the Western Pacific, Geophys. Res. Lett. 16, 527-530.

Levshin, A. L., Pisarenko, V. F., and Pogrebinsky, G. A. (1972), On a Frequency-time Analysis of Oscillations, Ann. Geophys. 28, 211-218.

Levshin, A. L., Ratnikova, L., and Berger, J. (1992), Peculiarities of Surface-wave Propagation across Central Eurasia, Bull. Seismol. Soc. Am. 82, 2464-2493.

McGarr, A. (1969), Amplitude Variations of Rayleigh Waves-Horizontal Refraction, Bull. Seismol. Soc. Am. 59, 1307-1334.

Melbourne, T. and Helmberger, D. (2001), Mantle Control on Plate Boundary Deformation, Geophys. Res. Lett. 28, 4003-4006.

Mitchell, B. J. (1995), Anelastic Structure and Evolution of the Continental Crust and Upper Mantle from Seismic Surface Wave Attenuation, Rev. of Geophys. 33, 441-462.

Montalbetti, J. F. and Kanasewich, E. R. (1970), Enhancement of Teleseismic Body Phases with a Polarization Filter, Geophys. J. R. astr. Soc. 21, 119-129.

Papoulis, A. Probability, Random Variables, and Stochastic Processes, 2nd edition (McGraw-Hill, New York. 1984).

Park, J. and Chave, A. D. (1984), Om the Estimation of Magnetelluric Response Functions Using the Singular Value Decomposition, Geophys. J. R. astr. Soc. 77, 683-709.

PARK, J. and Yu, Y. (1992), Anisotropy and Coupled Free Oscillations: Simplified Models and Surface Wave Observations, Geophys. J. Int. 110, 401-420.

PARK, J. and Yu, Y. (1993), Seismic Determination of Elastic Anisotropy and Mantle Flow, Science 261, 1159-1162.

Park, J., Vernon III, F. L., and Lindberg, C. R. (1987), Frequency-dependent Polarization Analysis of High-frequency Seismograms, J. Geophys. Res. 92, 12,664-12,674.

Patton, H. (1980), Crust and Upper Mantle Structure of the Eurasian Continent from the Phase Velocity and $Q$ of Surface Waves, Rev. Geophys. Space Phys. 18, 605-625.

Regan, J. and Anderson, D. L. (1984), Anisotropic Models of the Upper Mantle, Phys. Earth Planet. Inter., 35, 227-263.

Samson, J. C. (1983), Pure States, Polarized Waves, and Principal Components in the Spectra of Multiple, Geophysical Time-series, Geophys. J. R. astr. Soc. 72, 647-664.

Samson, J. C. and Olson, J. V. (1980), Some Comments on the Descriptions of the Polarization States of Waves, Geophys. J. R. astr. Soc. 61, 115-129.

Silver, P. G. and Chan, W. W. (1988), Implications for Continental Structure and Evolution from Seismic Anisotropy, Nature 335, 34-39.

Stewart, R. C. and Douglas, A. (1983), Seismograms from Phaseless Seismographs, Geophys. J. R. astr. Soc. 73, 517-521.

Vidale, J. E. (1986), Complex Polarization Analysis of Particle Motion, Bull. Seismol. Soc. Am. 76, 13931405.

Vig, P. K. and Mitchell, B. J. (1990), Anisotropy beneath Hawaii from Surface Wave Particle Motion Observations, Pure Appl. Geophys. 133, 1-22.

Vinnik, L. P., Veronique, F., and Romanowicz, B. (1989), Azimuthal Anisotropy in the Earth from Observations of SKS at GEOSCOPE and NARS Broadband Stations, Bull. Seismol. Soc. Am. 79, 15421558.

Woodhouse, J. H. and Wong, Y. K. (1986), Amplitude, Phase and Path Anomalies of Mantle Waves, Geophys. J. R. astr. Soc. 87, 753-773.

Yu, Y. and PARK, J. (1993), Upper Mantle Anisotropy and Coupled-mode Long-period Surface Waves, Geophys. J. Int. 114, 473-489. 
Yu, Y. and Park, J. (1994), Hunting for Azimuthal Anisotropy beneath the Pacific Ocean Region, J. Geophys. Res. 99, 15,399-15,421.

Zhang, J. (1998), Inversion of Surface Wave Spectra for Source Parameters of Large Earthquakes Using Aspherical Earth Models, Phys. Earth Planet. Inter. 107, 327-350.

(Received October 3, 2001, accepted February 9, 2002)

$$
\begin{aligned}
& \text { (D) To access this journal online: } \\
& \text { (20) http://www.birkhauser.ch }
\end{aligned}
$$

